\title{
MULTINORMED $W^{*}$-ALGEBRAS AND UNBOUNDED OPERATORS
}

\author{
ANAR DOSI \\ (Communicated by Marius Junge)
}

\begin{abstract}
In this paper we investigate multinormed $W^{*}$-algebras in terms of the central topologies of $W^{*}$-algebras. The main result asserts that each multinormed $W^{*}$-algebra can be realized as a local von Neumann algebra on a certain domain in a Hilbert space. Moreover, it admits the predual (unique up to an isometry), which is the $\ell_{1}$-normed space. In the normed case the assertion is reduced to the known Sakai theorem.
\end{abstract}

\section{INTRODUCTION}

The theory of $*$-algebras of unbounded operators in a Hilbert space (or briefly, $\mathrm{O}^{*}$-algebras) has great importance in many problems of the representation theory of Lie groups, quantum field theory and statistical physics. A survey of results on $\mathrm{O}^{*}$-algebras has been reflected in the monograph [28] by Schmüdgen. An $\mathrm{O}^{*}$ algebra is treated as a unital *-algebra $A$ of linear transformations on a common dense subspace $\mathcal{D}$ of a Hilbert space $H$ which leaves invariant $\mathcal{D}$. Each $T \in A$ being an unbounded operator on $H$ admits an unbounded dual $T^{\star}$ which leaves invariant $\mathcal{D}$; therefore $T^{*}=T^{\star} \mid \mathcal{D} \in A$ and the correspondence $T \mapsto T^{*}$ turns out to be a natural involution on $A$. Thus an $\mathrm{O}^{*}$-algebra $A$ on a dense subspace $\mathcal{D}$ is the $*$-subalgebra of the enveloping $\mathrm{O}^{*}$-algebra

$$
L^{*}(\mathcal{D})=\left\{T \in L(\mathcal{D}): \mathcal{D} \subseteq \operatorname{dom}\left(T^{\star}\right), T^{\star}(\mathcal{D}) \subseteq \mathcal{D}\right\} \quad \text { on } \quad \mathcal{D},
$$

where $L(\mathcal{D})$ is the algebra of all linear transformations on the space $\mathcal{D}$. The algebra $L^{*}(\mathcal{D})$ can be equipped with various polynormed (or locally convex) topologies (see [28, 3.3]) generalizing the well-known operator topologies in $\mathcal{B}(H)$. For example, the seminorms $s_{x, y}(T)=|\langle T x, y\rangle|, x, y \in \mathcal{D}$, and $s_{x}(T)=\|T x\|, x \in \mathcal{D}$, define the weak and strong operator topologies in $L^{*}(\mathcal{D})$, respectively. But the uniform operator topology has some diversity in this context (see [28, Ch. 4], [19, Ch. I]), which depends on the considered $\mathrm{O}^{*}$-algebra on $\mathcal{D}$. A $*$-representation of a unital *-algebra is defined as a $*$-homomorphism into $L^{*}(\mathcal{D})$ for a certain domain $\mathcal{D}$. The concepts of a generalized vector, standard systems and modular systems in this framework allow us to develop the Tomita-Takesaki theory of $\mathrm{O}^{*}$-algebras with its physical applications [19]. Further developments of these ideas, namely considering partial $\mathrm{O}^{*}$-algebras and their representations, may be found in [1.

Received by the editors March 15, 2010 and, in revised form, May 18, 2011.

2010 Mathematics Subject Classification. Primary 46K10; Secondary 47L25, 47L60.

Key words and phrases. Multinormed $W^{*}$-algebra, unbounded operators, central topology, $\ell_{1}$-normed space.

The author thanks the institution TUBITAK for encouraging research papers in Turkey. 
Another link to the theory of $\mathrm{O}^{*}$-algebras has been observed in the realization problem of an abstract quantum (or local operator) space. The representation theorem [5] for quantum spaces asserts that each quantum space $V$ can be realized (up to a topological matrix isomorphism) as a concrete quantum space on a quantum domain $\mathcal{E}$. This result generalizes the representation theorem for multinormed (or locally) $C^{*}$-algebras proved in 18 by Inoue. By a quantum domain in a Hilbert space $H$ we mean an upward filtered family $\mathcal{E}=\left\{H_{\alpha}: \alpha \in \Lambda\right\}$ of its closed subspaces whose union space $\mathcal{D}=\bigcup \mathcal{E}$ is dense in $H$. Equivalently, a quantum domain is defined by means of an upward directed family of projections $\mathcal{E}=\left\{p_{\alpha}: \alpha \in \Lambda\right\}$ in $\mathcal{B}(H)$ such that $\vee_{\alpha} p_{\alpha}=1_{H}$, where $\vee_{\alpha} p_{\alpha}$ is the least upper bound in $\mathcal{B}(H)$ of the projection net. The algebra of all noncommutative continuous functions on $\mathcal{D}$ is defined [5, 6], 7] as the $\mathrm{O}^{*}$-subalgebra

$$
C_{\mathcal{E}}^{*}(\mathcal{D})=\left\{T \in L(\mathcal{D}): p_{\alpha} T \subseteq T p_{\alpha}, T p_{\alpha} \in \mathcal{B}(H), \alpha \in \Lambda\right\} \subseteq L^{*}(\mathcal{D})
$$

The *-algebra $C_{\mathcal{E}}^{*}(\mathcal{D})$ possesses apart from $L^{*}(\mathcal{D})$ the canonical polynormed topology defined by means of the family $\|T\|_{\alpha}=\left\|T p_{\alpha}\right\|, T \in C_{\mathcal{E}}^{*}(\mathcal{D}), \alpha \in \Lambda$ of $C^{*}$ seminorms, which results in a unital multinormed $C^{*}$-algebra structure on it. This topology represents the operator norm topology in the locally convex space context. The representation theorem asserts that if $A$ is a unital multinormed $C^{*}$-algebra with its upward filtered family $\left\{\|\cdot\|_{\alpha}\right\}$ of $C^{*}$-seminorms, then there is a quantum domain $\mathcal{E}=\left\{p_{\alpha}\right\}$ and $*$-homomorphism $\varphi: A \rightarrow C_{\mathcal{E}}^{*}(\mathcal{D})$ such that $\|\varphi(a)\|_{\alpha}=\|a\|_{\alpha}$, $a \in A$ for all $\alpha$ (see [18, [5]). A similar result for multinormed $W^{*}$-algebras was investigated in [13 by Fragoulopoulou. A multinormed $W^{*}$-algebra is defined as the inverse limit of $W^{*}$-algebras with its weak* continuous connecting *-homomorphisms. The representation theorem from [13] asserts that each multinormed $W^{*}$-algebra is topologically $*$-isomorphic to a strongly closed $*$-subalgebra in $C_{\mathcal{E}}^{*}(\mathcal{D})$. This type of realization of a multinormed $W^{*}$-algebra would lead in to the locally convex version of von Neumann algebras to be defined as strongly closed *-subalgebras in $C_{\mathcal{E}}^{*}(\mathcal{D})$ (see [20, 21]). But this type of motivation needs to be done with more analysis than a direct comparison with respect to the normed case. For instance, we do not know whether the algebra $C_{\mathcal{E}}^{*}(\mathcal{D})$ indeed plays the role of $\mathcal{B}(H)$ in the locally convex space theory. Recent investigations in the duality theory for quantum spaces indicate that is not indeed the case. The bicommutant property fails to be true for strongly closed unital *-subalgebras in $C_{\mathcal{E}}^{*}(\mathcal{D})$ (see Example in Subsection 3.2). This property in its general setting has been investigated for unbounded operators in [2]. As shown in [10, the algebra $C_{\mathcal{E}}^{*}(\mathcal{D})$ has a well duality behavior if $\mathcal{E}$ admits a gradation, that is, each $p_{\alpha}=\sum_{\kappa \in \alpha} e_{\kappa}$ is a finite sum of an orthogonal family $\left(e_{\kappa}\right)$ of projections such that $\sum_{\kappa} e_{\kappa}=1$. In this case a quantum space version of the operator space $\mathcal{T}(H)$ of all trace class operators on a Hilbert space $H$ can be constructed using the inductive limit of operator spaces. Namely, $\mathcal{T}_{\mathcal{E}}(\mathcal{D})=\operatorname{op} \bigoplus_{\kappa} \mathcal{T}\left(e_{\kappa}(H)\right)$ is the quantum direct sum of the operator subspaces $\mathcal{T}\left(e_{\kappa}(H)\right) \subseteq \mathcal{T}(H)$. If $\mathcal{T}_{\mathcal{E}}(\mathcal{D})_{\beta}^{\prime}$ is the strong quantum dual (see [1]) of $\mathcal{T}_{\mathcal{E}}(\mathcal{D})$, then $\mathcal{T}_{\mathcal{E}}(\mathcal{D})_{\beta}^{\prime}=C_{\mathcal{E}}^{*}(\mathcal{D})$ up to a (canonical) topological matrix isomorphism. We could not expect a similar property for strongly closed $*$-subalgebras of $C_{\mathcal{E}}^{*}(\mathcal{D})$ in the general case. Actually, we do not know a possible link between the multinormed $W^{*}$-algebras, $*$-subalgebras in $C_{\mathcal{E}}^{*}(\mathcal{D})$ which admit preduals, and so-called locally von Neumann algebras. 
The present paper is devoted to filling these gaps of the theory. We propose another approach to the problem by investigating the multinormed completions of a $W^{*}$-algebra. If $B$ is a $W^{*}$-algebra and $\left(e_{\iota}\right)$ is a family of its central projections with $\vee_{\iota} e_{\iota}=1$, then we have a multinormed (or locally multiplicative convex) topology with its defining family of $C^{*}$-seminorms $\|b\|_{\iota}=\left\|b e_{\iota}\right\|, b \in B$ called $a$ central topology in $B$. It turns out (see Proposition 2.1) that the multinormed $W^{*}$-algebras are precisely the central completions of $W^{*}$-algebras. Furthermore, we prove that a multinormed $W^{*}$-algebra $A$ admits a unique (up to isometry) predual $X$ (in the bornological sense), which is an $\ell_{1}$-normed space (see Definition 2.1). In the normed case this fact is well known [25, 1.13.3]. Further, we focus on the operator $*$-representations of a multinormed $W^{*}$-algebra. As a locally convex version of $\mathcal{B}(H)$ we declare the $*$-algebra $C_{\mathcal{E}}^{*}(\mathcal{D})$ of all noncommutative continuous functions over a commutative domain $\mathcal{E}=\left\{p_{\alpha}: \alpha \in \Lambda\right\}$, that is, $\mathcal{E}$ is a mutually commuting family of projections in $\mathcal{B}(H)$. In this case $C_{\mathcal{E}}^{*}(\mathcal{D})$ is (see Lemma 3.1) a multinormed $W^{*}$-algebra. Actually, it is a central completion of the commutant $\mathcal{E}^{\prime}$ in $\mathcal{B}(H)$. In particular, it admits the unique predual, which is $\mathcal{T}_{\mathcal{E}}(\mathcal{D})$, whenever $\mathcal{E}$ admits a gradation. The representation theorem for multinormed $C^{*}$-algebras mentioned above is generalized in the following way. If $A$ is a multinormed $C^{*}$ algebra with its upward filtered family of $C^{*}$-seminorms $\left\{\|\cdot\|_{\alpha}\right\}$, then there is a (commutative) domain $\mathcal{E}=\left\{e_{\alpha}: \alpha \in \Lambda\right\}$ in a Hilbert space $H$ and $*$-embedding $\varphi: A \hookrightarrow C_{\mathcal{E}}^{*}(\mathcal{D})$ such that $\|\varphi(a)\|_{\alpha}=\|a\|_{\alpha}, a \in A, \alpha \in \Lambda$, where $\mathcal{D}$ is the union space of $\mathcal{E}$. A strongly closed $*$-subalgebra $A \subseteq C_{\mathcal{E}}^{*}(\mathcal{D})$ is said to be a local von Neumann algebra on $\mathcal{D}$ if $A p_{\alpha} \subseteq A$ for all $\alpha$. In this case, $A p_{\alpha}$ is a $W^{*}$-algebra and $A$ turns out to be an inductive limit of these $W^{*}$-algebras (see Proposition 3.2). For instance, the center $Z\left(C_{\mathcal{E}}^{*}(\mathcal{D})\right)$ of $C_{\mathcal{E}}^{*}(\mathcal{D})$ is a local von Neumann algebra on $\mathcal{D}$, which is the strong closure of the unital associative subalgebra generated by $\mathcal{E}$. As the main result, we prove a Sakai (see [25, 1.16.7] for the normed case) type theorem for multinormed $W^{*}$-algebras. Namely, if $A$ is a multinormed $W^{*}$-algebra with its upward filtered family $\left\{\|\cdot\|_{\alpha}: \alpha \in \Lambda\right\}$ of $C^{*}$-seminorms, then there exists a domain $\mathcal{E}=\left\{p_{\alpha}: \alpha \in \Lambda\right\}$ in a Hilbert space $H$ and a $*$-isomorphism $A \rightarrow C_{\mathcal{E}}^{*}(\mathcal{D})$ onto a local von Neumann subalgebra on $\mathcal{D}$ such that $\|a\|_{\alpha}=\left\|a p_{\alpha}\right\|, a \in A$ for all $\alpha$. Thus the multinormed $W^{*}$-algebras are precisely $*$-subalgebras in $C_{\mathcal{E}}^{*}(\mathcal{D})$ which admit preduals, and they are in turn the local von Neumann algebras.

As an interesting application of local operator algebras from $C_{\mathcal{E}}^{*}(\mathcal{D})$ to the quantum moment problems we refer the reader to [7].

\section{The Predual of A MUltinormed $W^{*}$-Algebra}

In this section we investigate the central completions of a $W^{*}$-algebra determined by means of its central projections. We prove that each multinormed $W^{*}$-algebra is the bornological dual of the uniquely (up to an isometry) defined $\ell_{1}$-normed space.

2.1. The central topology. Let $A$ be a $*$-algebra. A seminorm $p$ on $A$ is said to be a $C^{*}$-seminorm if $p\left(a^{*} a\right)=p(a)^{2}$ for all $a \in A$. It turns out (see [14, 7.2]) that each $C^{*}$-seminorm preserves the involution $*$ on $A$, and it is submultiplicative. A complete polynormed (or locally convex) algebra $A$ whose topology is defined by a (separated) family of $C^{*}$-seminorms $\left\{\|\cdot\|_{\iota}: \iota \in \Xi\right\}$ is called a multinormed (or locally) $C^{*}$-algebra. In particular, it is an Arens-Michael algebra whose decomposition [16, 5.2.10] allows us to represent it as an inverse limit $A=\lim \left\{A_{\iota}, \varphi_{\iota \kappa}\right\}$ 
of $C^{*}$-algebras $A_{\iota}$ such that all connecting maps $\varphi_{\iota \kappa}: A_{\kappa} \rightarrow A_{\iota}(\iota \leq \kappa)$ are *homomorphisms.

Now let $A$ be a multinormed $C^{*}$-algebra with its defining family of $C^{*}$-seminorms $\left\{\|\cdot\|_{\iota}: \iota \in \Xi\right\}$ and let $B=\mathfrak{b}(A)$ be the set of all bounded elements in $A$, that is, $\sup _{\iota}\|b\|_{\iota}<\infty$. Then $B$ is a $C^{*}$-algebra equipped with the norm $\|\cdot\|=\sup _{\iota}\|\cdot\|_{\iota}$ (see [24). For brevity we say that $\left(A,\left\{\|\cdot\|_{\iota}\right\}\right)$ is a multinormed $C^{*}$-algebra. Put $J_{\iota}=\operatorname{ker}\|\cdot\|_{\iota}$ and $I_{\iota}=B \cap J_{\iota}$. Let us also accept the notation $\Lambda$ along with the index set $\Xi$ to indicate the set of all its finite subsets. So, $\alpha \in \Lambda$ means that $\alpha \subseteq \Xi$ is a finite subset. We will keep up this notation further throughout the paper.

Lemma 2.1. The ideal $I_{\iota}$ is closed in $B$ and $\|\cdot\|=\|\cdot\|_{\iota}$ on $B / I_{\iota}$. Moreover, $B / I_{\iota}=A / J_{\iota}$ up to a $*$-isomorphism.

Proof. If $b=\lim _{n} b_{n}$ in the $C^{*}$-algebra $B$ for a certain sequence $\left(b_{n}\right) \subseteq I_{\iota}$, then $\|b\|_{\iota} \leq\left\|b-b_{n}\right\|_{\iota}+\left\|b_{n}\right\|_{\iota} \leq\left\|b-b_{n}\right\|$, that is, $b \in I_{\iota}$. Thus $I_{\iota}$ is a closed ideal in $B$. In particular, it is selfadjoint and $\left(B / I_{\iota},\|\cdot\|\right)$ is a $C^{*}$-algebra too. Further, using [27, 5.4], we conclude that $A / J_{\iota}$ is a $C^{*}$-algebra with the quotient norm $\|\cdot\|_{\iota}$, and the quotient mapping $\pi_{\iota}: A \rightarrow A / J_{\iota}$ is a $*$-homomorphism. Since $B$ is dense in $A$, it follows that $\pi_{\iota}: B \rightarrow A / J_{\iota}$ has the dense range. Therefore $\pi_{\iota}(B)=A / J_{\iota}$ (see for instance [23, 3.1.5]). In particular, $B / I_{\iota}=A / J_{\iota}$ up to a $*$-isomorphism, where $B / I_{\iota}$ is equipped with the quotient $C^{*}$-norm $\|\cdot\|_{\iota}$. Thus we have the $C^{*}$-norms $\|\cdot\|$ and $\|\cdot\|_{\iota}$ on the $*$-algebra $B / I_{\iota}$. Hence $\|\cdot\|=\|\cdot\|_{\iota}$.

A weak* continuous *-homomorphism between $W^{*}$-algebras is called a $W^{*}$ homomorphism. By a multinormed $W^{*}$-algebra $A$ we mean an inverse limit $A=$ $\varliminf_{\iota}\left\{A_{\iota}, \varphi_{\iota \kappa}\right\}$ of $W^{*}$-algebras $A_{\iota}$ such that all connecting maps $\varphi_{\iota \kappa}: A_{\kappa} \rightarrow A_{\iota}$ $(\iota \leq \kappa)$ are $W^{*}$-homomorphisms (Fragoulopoulou [13]). We left the terminology locally $W^{*}$-algebra for an inverse limit of $W^{*}$-algebras. If $\|a\|_{\iota}=\left\|\varphi_{\iota}(a)\right\|, a \in A$, then $\left\{\|\cdot\|_{\iota}\right\}$ is a defining family of $C^{*}$-seminorms on $A$, where $\varphi_{\iota}: A \rightarrow A_{\iota}\left(A_{\iota}=A / J_{\iota}\right)$ is the canonical projection.

Lemma 2.2. If $\left(A,\left\{\|\cdot\|_{\iota}\right\}\right)$ is a multinormed $W^{*}$-algebra and $B$ is the $C^{*}$-algebra of all its bounded elements, then $B$ is a $W^{*}$-algebra and all ideals $I_{\iota}$ are weak* closed in $B$.

Proof. First note that $A_{\iota}=A / J_{\iota}=B / I_{\iota}$ thanks to Lemma 2.1. So, each $B / I_{\iota}$ is a $W^{*}$-algebra and let $Y_{\iota}$ be its predual. If $\mathfrak{B}=\bigoplus_{\iota}^{\infty} B / I_{\iota}\left(\ell_{\infty}\right.$-direct sum $)$ is the direct sum of $W^{*}$-algebras, then $\mathfrak{B}$ is a $W^{*}$-algebra with its predual $Y=\bigoplus_{\iota}^{1} Y_{\iota}\left(\ell_{1^{-}}\right.$ direct sum). Consider the $*$-homomorphism $\varphi: B \rightarrow \mathfrak{B}, \varphi(b)=\left(\varphi_{\iota}(b)\right)_{\iota}$. Using Lemma 2.1, we derive that

$$
\|b\|=\sup _{\iota}\|b\|_{\iota}=\sup _{\iota}\left\|\varphi_{\iota}(b)\right\|_{\iota}=\sup _{\iota}\left\|\varphi_{\iota}(b)\right\|=\|\varphi(b)\|
$$

for all $b \in B$, that is, $\varphi$ is an isometry. Actually $\varphi(B)$ is weak* closed in $\mathfrak{B}$. Indeed, take a net $\left(\varphi\left(b_{\lambda}\right)\right)$ in $\varphi(B)$ such that $u=\left(u_{\iota}\right)_{\iota}=w^{*} \lim _{\lambda} \varphi\left(b_{\lambda}\right) \in \mathfrak{B}$. If $\prod_{\iota} \sigma\left(B / I_{\iota}, Y_{\iota}\right)$ is the direct product of weak ${ }^{*}$ topologies in $\prod_{\iota} B / I_{\iota}$ and $\bigoplus_{\iota} Y_{\iota}$ is the direct algebraic sum, then $\prod_{\iota} \sigma\left(B / I_{\iota}, Y_{\iota}\right)=\sigma\left(\prod_{\iota} B / I_{\iota}, \bigoplus_{\iota} Y_{\iota}\right)$ (see [26, 4.4, Corollary 1]), where $\sigma$ indicates the weak topology of the relevant dual pairs. Hence 
$\left(\prod_{\iota} \sigma\left(B / I_{\iota}, Y_{\iota}\right)\right) \mid \mathfrak{B}=\sigma\left(\mathfrak{B}, \bigoplus_{\iota} Y_{\iota}\right) \subseteq \sigma(\mathfrak{B}, Y)$. It follows that $u_{\iota}=w^{*} \lim _{\lambda} \varphi_{\iota}\left(b_{\lambda}\right)$ in $B / I_{\iota}$ for each $\iota$. Assume $\iota \leq \kappa$. Taking into account that the connecting mapping $\varphi_{\iota \kappa}: A / J_{\kappa} \rightarrow A / J_{\iota}$ is a $W^{*}$-homomorphism, we conclude that

$$
u_{\iota}=w^{*} \lim _{\lambda} \varphi_{\iota}\left(b_{\lambda}\right)=w^{*} \lim _{\lambda} \varphi_{\iota \kappa} \varphi_{\kappa}\left(b_{\lambda}\right)=\varphi_{\iota \kappa}\left(w^{*} \lim _{\lambda} \varphi_{\kappa}\left(b_{\lambda}\right)\right)=\varphi_{\iota \kappa}\left(u_{\kappa}\right) .
$$

Hence $a=\left(u_{\iota}\right) \in \lim \left\{A_{\iota}, \varphi_{\iota \kappa}\right\}=A$ and $\varphi_{\iota}(a)=u_{\iota}$ for all $\iota$. Using again Lemma 2.1, we obtain that $\sup _{\iota}\|a\|_{\iota}=\sup _{\iota}\left\|\varphi_{\iota}(a)\right\|_{\iota}=\sup _{\iota}\left\|u_{\iota}\right\|=\|u\|_{\mathfrak{B}}<\infty$, that is, $a \in B$. So, $\varphi(a)=\left(\varphi_{\iota}(a)\right)_{\iota}=u \in \varphi(B)$. Hence $B$ is a $W^{*}$-algebra.

Finally, note that $\varphi_{\iota}: B \rightarrow B / I_{\iota}$ is a $W^{*}$-homomorphism, for $\varphi_{\iota}(b)\left(y_{\iota}\right)=$ $\varphi(b)\left(y_{\iota}\right)$ for all $y_{\iota} \in Y_{\iota}$ and $b \in B$. Hence $I_{\iota}=\operatorname{ker} \varphi_{\iota}$ is weak ${ }^{*}$ closed in $B$.

Now let $B$ be a $W^{*}$-algebra and let $\left(e_{\iota}\right)$ be a family of its central projections such that $\vee_{\iota} e_{\iota}=1$. For each $e_{\iota}$ we set $\|b\|_{\iota}=\left\|b e_{\iota}\right\|, b \in B$. Then $\|\cdot\|_{\iota}$ is a $C^{*}$-seminorm on $B$. Indeed, $\left\|b^{*} b\right\|_{\iota}=\left\|b^{*} b e_{\iota}\right\|=\left\|\left(b e_{\iota}\right)^{*} b e_{\iota}\right\|=\left\|b e_{\iota}\right\|^{2}=\|b\|_{\iota}^{2}, b \in B$.

Lemma 2.3. The following assertions are equivalent:

(i) $B e_{\iota} \subseteq B e_{\kappa}$,

(ii) $e_{\iota} \leq e_{\kappa}$,

(iii) $\|\cdot\|_{\iota} \leq C_{\iota \kappa}\|\cdot\|_{\kappa}$ for a certain constant $C_{\iota \kappa}>0$.

Moreover, if $e_{\alpha}=\vee_{\iota \in \alpha} e_{\iota}$ for a finite subset $\left\{e_{\iota}: \iota \in \alpha\right\}$ and $\|b\|_{\alpha}=\left\|b e_{\alpha}\right\|, b \in B$ is the relevant $C^{*}$-seminorm on $B$, then $\|\cdot\|_{\alpha}=\max _{\iota \in \alpha}\|\cdot\|_{\iota}$.

Proof. $(i) \Rightarrow(i i)$. If $e_{\iota}=b e_{\kappa}$ for a certain $b \in B$, then $e_{\iota} e_{\kappa}=b e_{\kappa}=e_{\iota}$, that is, $e_{\iota} \leq e_{\kappa}$.

$(i i) \Rightarrow(i i i)$. If $e_{\iota} \leq e_{\kappa}$, then $\|b\|_{\iota}^{2}=\left\|b^{*} b\right\|_{\iota}=\left\|b^{*} e_{\iota} b\right\| \leq\left\|b^{*} e_{\kappa} b\right\|=\|b\|_{\kappa}^{2}$ for all $b \in B$.

$($ iii $) \Rightarrow(i)$. If $\|\cdot\|_{\iota} \leq C_{\iota \kappa}\|\cdot\|_{\kappa}$ and $b \in B$, then $\left\|b\left(1-e_{\kappa}\right)\right\|_{\iota} \leq C_{\iota \kappa}\left\|b\left(1-e_{\kappa}\right)\right\|_{\kappa}$ $=0$, that is, $b\left(1-e_{\kappa}\right) e_{\iota}=0$. Then $b e_{\iota}=b e_{\iota} e_{\kappa} \in B e_{\kappa}$, that is, $B e_{\iota} \subseteq B e_{\kappa}$.

Further, if $\vee_{\iota \in \alpha} B e_{\iota}$ is the weak ${ }^{*}$ closed ideal generated by a finite subset $\left\{e_{\iota}\right.$ : $\iota \in \alpha\}$, then $\vee_{\iota \in \alpha} B e_{\iota}=B e_{\alpha}$, where $e_{\alpha}=\vee_{\iota \in \alpha} e_{\iota}$. Indeed, $\vee_{\iota \in \alpha} B e_{\iota}=B p$ for a certain central projection $p \in B$ [25, 1.10.5]. Then $p \geq \vee_{\iota \in \alpha} e_{\iota}$, for $B e_{\iota} \subseteq B p$, $\iota \in \alpha$. But $B e_{\alpha}$ is a weak* closed ideal containing all $B e_{\iota}$. Hence $B e_{\alpha} \supseteq B p$ and $\vee_{\iota \in \alpha} e_{\iota}=e_{\alpha} \geq p$, that is, $e_{\alpha}=p$ and $\|\cdot\|_{\alpha} \geq \max _{\iota \in \alpha}\|\cdot\|_{\iota}$. Conversely, using the Sakai theorem [25, 1.16.7], one can assume that $B$ is a von Neumann algebra on a Hilbert space $H$ and $e_{\alpha}=1$. We can also assume that we have been dealing with two central projections $e_{1}$ and $e_{2}$ of $B$ such that $e_{1} \vee e_{2}=1$. If $f_{i}=1-e_{i}$, then $H=$ $e_{1} e_{2}(H) \oplus f_{2} e_{1}(H) \oplus f_{1} e_{2}(H) \oplus f_{1} f_{2}(H)$. Moreover, this orthogonal decomposition reduces each $b \in B$. If $x \in f_{1} f_{2}(H)$, then $x \perp e_{1}(H)$ and $x \perp e_{2}(H)$, that is, $x \perp\left(e_{1}(H)+e_{2}(H)\right)$. But $e_{1}(H)+e_{2}(H)$ is dense in $H$, for $e_{1} \vee e_{2}=1$. Hence $x=0$. Thus $f_{1} f_{2}(H)=\{0\}$ or $H=e_{1} e_{2}(H) \oplus f_{2} e_{1}(H) \oplus f_{1} e_{2}(H)$. It follows that $\|b\|=\max \left\{\left\|b e_{1} e_{2}\right\|,\left\|b f_{2} e_{1}\right\|,\left\|b f_{1} e_{2}\right\|\right\} \leq \max \left\{\left\|b e_{1}\right\|,\left\|b e_{2}\right\|\right\}$. Consequently, $\|\cdot\|_{\alpha} \leq \max _{\iota \in \alpha}\|\cdot\|_{\iota}$.

The family $\left\{\|\cdot\|_{\iota}\right\}$ of $C^{*}$-seminorms on a $W^{*}$-algebra $B$ associated with central projections $\left(e_{\iota}\right), \vee_{\iota} e_{\iota}=1$, defines a new polynormed topology in $B$ called a central topology. Note that each central topology is Hausdorff. If $\|b\|_{\iota}=0$ for all $\iota$, then for each finite index set $\alpha$, we have $b e_{\alpha}=0$ by Lemma 2.3. Since $\vee_{\alpha} e_{\alpha}=1$, it follows that $b=w^{*} \lim _{\alpha} b e_{\alpha}=0$. Furthermore, based on Lemma 2.3 we assert that a central topology can also be defined by means of an upward filtered family 
of projections $\left(e_{\alpha}\right)$. Two upward filtered families $\left(e_{\alpha}\right)$ and $\left(f_{v}\right)$ of projections in $B$ are assumed to be equivalent (and we write $\left(e_{\alpha}\right) \sim\left(f_{v}\right)$ ) if for each $e_{\alpha}$ there corresponds $f_{v}$ such that $e_{\alpha} \leq f_{v}$ and vice versa.

Corollary 2.1. Let $B$ be a $W^{*}$-algebra with upward filtered families $\left(e_{\alpha}\right)$ and $\left(f_{v}\right)$ of projections such that $\vee_{\alpha} e_{\alpha}=\vee_{v} f_{v}=1$. They define the same central topology in $B$ iff $\left(e_{\alpha}\right) \sim\left(f_{v}\right)$.

Proof. The families $\left(e_{\alpha}\right)$ and $\left(f_{v}\right)$ define the same central topology iff for each $e_{\alpha}$ there corresponds $f_{v}$ such that $\|\cdot\|_{\alpha} \leq C_{\alpha v}\|\cdot\|_{v}$, and vice versa. It remains to use Lemma 2.3 .

Thus there is a one-to-one correspondence between central topologies on a $W^{*}$ algebra $B$ and the equivalent classes of upward filtered to 1 central projections in $B$. The completion of $B$ with respect to a central topology turns out to be a multinormed $C^{*}$-algebra. We say that it is a central completion of $B$.

Proposition 2.1. Let $A$ be a multinormed $C^{*}$-algebra. The algebra $A$ is a multinormed $W^{*}$-algebra if and only if it is a central completion of a $W^{*}$-algebra.

Proof. First assume that $\left(A,\left\{\|\cdot\|_{\iota}\right\}\right)$ is a multinormed $W^{*}$-algebra. By Lemma 2.2 , the $C^{*}$-algebra $B$ of all its bounded elements turns out to be a $W^{*}$-algebra with the weakly closed ideals $I_{\iota}$. Then $I_{\iota}=B f_{\iota}$ for a certain central projection $f_{\iota} \in B$ [25, 1.10.5]. Put $e_{\iota}=1-f_{\iota}$. Since $B \wedge_{\iota} f_{\iota}=\bigcap_{\iota} B f_{\iota}=\bigcap_{\iota} I_{\iota}=\{0\}$, it follows that $\vee_{\iota} e_{\iota}=1-\wedge_{\iota} f_{\iota}=1$. The restriction of the mapping $\varphi_{\iota}: B \rightarrow B / I_{\iota}$ to $B e_{\iota}$ is a *-isomorphism; therefore it is an isometry. Using Lemma 2.1, we obtain that

$$
\|b\|_{\iota}=\left\|\varphi_{\iota}(b)\right\|_{\iota}=\left\|\varphi_{\iota}(b)\right\|=\left\|\varphi_{\iota}\left(b e_{\iota}\right)\right\|=\left\|b e_{\iota}\right\|
$$

for all $b \in B$. So, $\left\{\|\cdot\|_{\iota}\right\}$ is the defining family of $C^{*}$-seminorms of the central topology associated with the central projections $\left(e_{\iota}\right)$. It remains to note that $B$ is dense in the complete algebra $A$. Hence $A$ is a central completion of the $W^{*}$-algebra $B$.

Conversely, assume that $A$ is a central completion of a $W^{*}$-algebra $B$ associated with central projections $\left(e_{\iota}\right)$ of $B$ with $\vee_{\iota} e_{\iota}=1$. We write $\iota \leq \kappa$ whenever $e_{\iota} \leq e_{\kappa}$. The latter means that $\|\cdot\|_{\iota} \leq\|\cdot\|_{\kappa}$ thanks to Lemma 2.3. Put $A_{\iota}=B e_{\iota}$. If $\iota \leq \kappa$, then $\varphi_{\iota \kappa}: A_{\kappa} \rightarrow A_{\iota}, \varphi_{\iota \kappa}(x)=x e_{\iota}$ is a $W^{*}$-homomorphism [25, 1.7.7]. By Lemma 2.3. $\left\{A_{\iota}, \varphi_{\iota \kappa}\right\}$ is an inverse system and $C=\lim \left\{A_{\iota}, \varphi_{\iota \kappa}\right\}$ is a multinormed $W^{*}$-algebra. Obviously, $C$ is the central completion of $B$. Hence $A=C$ and $A$ is a multinormed $W^{*}$-algebra.

Corollary 2.2. If $A$ is a multinormed $W^{*}$-algebra such that $\mathfrak{b}(A)$ is a factor, then $A$ is a $W^{*}$-algebra.

If $B=\bigoplus_{\iota}^{\infty} B_{\iota}$ is an infinite direct sum of factors, then we have a canonical family $\left(e_{\iota}\right)$ of its central orthogonal projections in $B$ such that $\sum_{\iota} e_{\iota}=1$. If $A$ is the central completion of $B$ associated with this family, then $A$ is a multinormed $W^{*}$ algebra (Proposition 2.1). Actually, $A=\prod_{\iota} B_{\iota}$. Therefore $\mathfrak{b}(A)=B$. Obviously $B$ is not simple and $A$ is not a normed $C^{*}$-algebra (see Problem 4.17 from [15]). 
2.2. The $\ell_{1}$-normed spaces. Let $B$ be a $W^{*}$-algebra equipped with a central topology associated with central projections $\left(e_{\iota}\right)_{\iota \in \Xi}, \vee_{\iota} e_{\iota}=1$. As above $I_{\iota}=$ ker $\|\cdot\|_{\iota}$ is a closed ideal in $B$, and $I_{\alpha}=\operatorname{ker}\|\cdot\|_{\alpha}=\bigcap_{\iota \in \alpha} I_{\iota}$ for each $\alpha \in \Lambda$. If $Y$ is the predual of $B$, then we set $Y_{\iota}=Y \cap I_{\iota}^{\perp}$, where $I_{\iota}^{\perp} \subseteq Y^{* *}$ is the polar of the subspace $I_{\iota} \subseteq Y^{*}$ with respect to the dual pair $\left(Y^{*}, Y^{* *}\right)$. Thus $Y_{\iota}$ is the polar of $I_{\iota}$ with respect to the dual pair $\left(Y^{*}, Y\right)$. Namely, $Y_{\iota}=\left\{y \in Y: y^{\prime}\left(I_{\iota}\right)=\{0\}\right\}$ is a closed subspace in $Y$, where $y^{\prime}(f)=f(y), f \in Y^{*}$. In particular, $Y_{\alpha}=$ $Y \cap I_{\alpha}^{\perp}=\left(\sum_{\iota \in \alpha} Y_{\iota}\right)^{-}$is the closure in $Y$ thanks to the bipolar theorem (see [26. 4.1, Corollary 2]) and the Mazur theorem [22, 10.4.9].

Lemma 2.4. For each $b \in B$ we have $\left\|b\left|Y_{\alpha}\|=\| b\left\|_{\alpha}=\max _{\iota \in \alpha}\right\| b\right| Y_{\iota}\right\|$. In particular, $Y_{\alpha}$ is a quotient of $\bigoplus_{\iota \in \alpha}^{1} Y_{\iota}$.

Proof. First let us prove that $\left\|b \mid Y_{\iota}\right\|=\|b\|_{\iota}$. By its very definition, $\left\|b \mid Y_{\iota}\right\|=$ $\sup \mid b\left(\right.$ ball $\left.Y_{\iota}\right) \mid=\sup \left\{\left|y^{\prime}(b)\right|: y \in\right.$ ball $\left.Y_{\iota}\right\}$. Take $y \in$ ball $Y_{\iota} \backslash\{0\}$. Then $y^{\prime}\left(I_{\iota}\right)=$ $\{0\}$. Therefore $y^{\prime}=\bar{y} \pi_{\iota}$ for the uniquely defined $\bar{y} \in$ ball $\left(B / I_{\iota}\right)^{*}$, where $\pi_{\iota}$ : $B \rightarrow B / I_{\iota}$ is the quotient homomorphism. If ball $\|\cdot\|_{\iota}$ (respectively, ball $\|\cdot\|$ ) is the unit set in $B$ to the $C^{*}$-seminorm $\|\cdot\|_{\iota}$ (respectively, original $C^{*}$-norm), then using Lemma 2.1, we derive that

$$
\begin{aligned}
\sup \mid y^{\prime}\left(\text { ball }\|\cdot\|_{\iota}\right) \mid & =\sup \mid \bar{y}\left(\text { ball }\|\cdot\|_{\iota}\right)|=\sup | \bar{y}(\text { ball }\|\cdot\|) \mid \\
& =\sup \mid y^{\prime}(\text { ball }\|\cdot\|) \mid=\left\|y^{\prime}\right\|=\|y\| \leq 1 .
\end{aligned}
$$

We have used the same notation ball $\|\cdot\|_{\iota}$ (respectively, ball $\left.\|\cdot\|\right)$ for the unit set to the quotient norm $\|\cdot\|_{\iota}$ (respectively, $\|\cdot\|$ ) on $B / I_{\iota}$. Therefore, if $\|b\|_{\iota}>0$, then $\left|y^{\prime}(b)\right|=\left|y^{\prime}\left(\|b\|_{\iota}^{-1} b\right)\right|\|b\|_{\iota} \leq\|b\|_{\iota}$. If $\|b\|_{\iota}=0$, then $b \in I_{\iota}$ and $y^{\prime}(b)=0$. Thus $\left\|b \mid Y_{\iota}\right\| \leq\|b\|_{\iota}$.

Conversely, assume that $\|b\|_{\iota}>0$. Using Lemma 2.1. we conclude that $\|b\|_{\iota}=$ $\left\|\pi_{\iota}(b)\right\|_{\iota}=\left\|\pi_{\iota}(b)\right\|$. By the Hahn-Banach extension theorem, $\left\|\pi_{\iota}(b)\right\|=\left|F^{\prime}\left(\pi_{\iota}(b)\right)\right|$ for some $F^{\prime} \in \operatorname{ball}\left(B / I_{\iota}\right)^{*}$. So, $\|b\|_{\iota}=|F(b)|$ for some $F \in$ ball $B^{*}, F\left(I_{\iota}\right)=\{0\}$, that is, $F \in$ ball $I_{\iota}^{\perp}$.

Let us prove that ball $Y_{\iota}$ is $\sigma\left(Y^{* *}, Y^{*}\right)$-dense in ball $I_{\iota}^{\perp}$. Note that $Y_{\iota}=I_{\iota}^{\perp}$ is the polar of $I_{\iota}$ with respect to the dual pair $\left(Y^{*}, Y\right)$, and its double polar $I_{\iota}^{\perp \perp}$ coincides with its $\sigma\left(Y^{*}, Y\right)$-closure. Using Proposition 2.1 and Lemma 2.2, we conclude that $I_{\iota}$ is weak ${ }^{*}$ closed; therefore $I_{\iota}^{\perp \perp}=I_{\iota}$ and the canonical identification $Y^{*} / I_{\iota}=Y_{\iota}^{*}$ turns out to be isometric (see [4, 5.2.3]). But $\left(Y^{*} / I_{\iota}\right)^{*}=I_{\iota}^{\perp}$ up to the isometry [4, 5.2.2]. Thus $Y_{\iota}^{* *}=I_{\iota}^{\perp}$. Now we use the well-known (see [4, 5.4.1]) fact that ball $Y_{\iota}$ is $\sigma\left(Y_{\iota}^{* *}, Y_{\iota}^{*}\right)$-dense in ball $Y_{\iota}^{* *}$. But

$$
\sigma\left(Y_{\iota}^{* *}, Y_{\iota}^{*}\right)=\sigma\left(I_{\iota}^{\perp}, Y^{*} / I_{\iota}\right)=\sigma\left(\left(Y^{*} / I_{\iota}\right)^{*}, Y^{*} / I_{\iota}\right)=\sigma\left(Y^{* *}, Y^{*}\right) \mid I_{\iota}^{\perp}
$$

(see [4, 5.2.2]). Hence ball $Y_{\iota}$ is $\sigma\left(Y^{* *}, Y^{*}\right)$-dense in ball $I_{\iota}^{\perp}$. In particular, $F=$ $\sigma\left(Y^{* *}, Y^{*}\right)-\lim _{\lambda} y_{\lambda}$ for a certain net $\left(y_{\lambda}\right)$ in ball $Y_{\iota}$. Then

$$
\|b\|_{\iota}=|F(b)|=\lim _{\lambda}\left|y_{\lambda}^{\prime}(b)\right|=\lim _{\lambda}\left|b\left(y_{\lambda}\right)\right| \leq \sup \mid b\left(\text { ball } Y_{\iota}\right)\left|=\left\|b \mid Y_{\iota}\right\| .\right.
$$

Consequently, $\left\|b \mid Y_{\iota}\right\|=\|b\|_{\iota}$.

Finally, consider the contraction $\varphi: \bigoplus_{\iota \in \alpha}^{1} Y_{\iota} \rightarrow Y_{\alpha}, \varphi\left(y_{\iota}\right)_{\iota}=\sum_{\iota \in \alpha} y_{\iota}$. Note that $Y_{\alpha}^{*}=B e_{\alpha}$. Indeed, $Y_{\alpha}^{\perp}=I_{\alpha}$ thanks to the bipolar theorem. It follows 
that the canonical isomorphism $B / I_{\alpha} \rightarrow Y_{\alpha}^{*}$ is an isometry. Hence $B e_{\alpha} \rightarrow Y_{\alpha}^{*}$, $a \mapsto a \mid Y_{\alpha}$ is an isometric isomorphism. If $\varphi^{*}: Y_{\alpha}^{*} \rightarrow \bigoplus_{\iota \in \alpha}^{\infty} Y_{\iota}^{*}, \varphi^{*}(b)=\left(b \mid Y_{\iota}\right)_{\iota}$ is the dual mapping, then $\left\|\varphi^{*}(b)\right\|=\max _{\iota \in \alpha}\left\|b \mid Y_{\iota}\right\|=\max _{\iota \in \alpha}\|b\|_{\iota}=\|b\|_{\alpha}$ thanks to Lemma 2.3. Thus $\varphi^{*}$ is an isometry. It follows that $\varphi$ is a quotient mapping, that is, $\varphi$ implements an isometric isomorphism of the quotient of $\bigoplus_{\iota \in \alpha}^{1} Y_{\iota} / \operatorname{ker}(\varphi)$ and the Banach space $Y_{\alpha}$.

Consider the subspace $X=\bigcup_{\alpha} Y_{\alpha}$ in $Y$, which possesses a natural complete bornology determined by the family $\left\{\right.$ ball $\left.Y_{\alpha}\right\}$. Note that $X$ is a dense subspace in $Y$. Indeed, if $b(X)=\{0\}$ for a certain $b \in B$, then $b \in Y_{\iota}^{\perp}$ for all $\iota$. But $Y_{\iota}^{\perp}=I_{\iota}$ (see the proof of Lemma 2.4). Then $\|b\|=\sup _{\iota}\|b\|_{\iota}=0$, that is, $b=0$. Hence $X$ is a dense subspace in $Y$. Further, using Lemma 2.4 and [26, 2.6.3], we derive that the complete bornological space $X$ is just the bornological quotient of the polynormed (or locally convex) direct sum $\bigoplus_{\iota} Y_{\iota}$. Note that $X=\bigoplus_{\iota} Y_{\iota}$ whenever $\left(e_{\iota}\right)$ is an orthogonal family of projections.

Definition 2.1. Let $X$ be a normed space. We say that $X$ is an $\ell_{1}$-normed space if it is a locally quotient $\pi: \bigoplus_{\iota \in \Xi} E_{\iota} \rightarrow X$ of the direct sum of some Banach spaces in the sense that $\pi$ is onto and for each $\alpha$ it is a quotient mapping of the finite $\ell_{1}$-sum $\bigoplus_{\iota \in \alpha}^{1} E_{\iota}$ onto its range.

If $Y_{\alpha}=\pi\left(\bigoplus_{\iota \in \alpha}^{1} E_{\iota}\right)$, then $Y_{\alpha}$ is complete in $X$ and $X=\bigcup_{\alpha} Y_{\alpha}$. In particular, $X$ is equipped with the bornology $\left\{\right.$ ball $\left.Y_{\alpha}\right\}$.

Examples: 1. The algebraic sum $X=\sum_{\iota} E_{\iota}$ in the $\ell_{1}$-sum $E=\bigoplus_{\iota}^{1} E_{\iota}$ of Banach spaces is an $\ell_{1}$-normed space. In this case $Y_{\alpha}=\bigoplus_{\iota \in \alpha}^{1} E_{\iota}$ for all $\alpha$.

2. Each Banach space is obviously an $\ell_{1}$-normed space.

3. If $V$ is a complete operator space, then it is a norm-completion of a certain $\ell_{1}$-normed space $X$. Indeed, there is a (matrix) quotient mapping $\pi: \bigoplus_{\iota}^{1} T_{n_{\iota}} \rightarrow V$, where $T_{n_{\iota}}$ is the $n_{\iota}$-squared trace class matrix algebra (see [3], [10]). If $X=$ $\pi\left(\sum_{\iota} T_{n_{\iota}}\right)$, then $X$ is an $\ell_{1}$-normed space, and it is dense in $V$.

4. The latter example can be generalized in the following way. Each $\ell_{1}$-normed quotient of the direct algebraic sum $\bigoplus_{\iota} E_{\iota}$ with its finite dimensional spaces $E_{\iota}$ is an $\ell_{1}$-normed space.

Now let $X$ be an $\ell_{1}$-normed space with its bornology $\left\{\right.$ ball $\left.Y_{\alpha}\right\}$. The bornological dual $X^{\prime}$ of $X$ [17] (see also [9]) is defined as the space of all linear functionals $f: X \rightarrow \mathbb{C}$ such that $d_{\alpha}(f)=\sup \mid f\left(\right.$ ball $\left.Y_{\alpha}\right) \mid<\infty$ for all $\alpha$. It turns out to be a polynormed space with its defining family of seminorms $\left\{d_{\alpha}\right\}$. This space can also be thought of as the dual space of the inductive limit $\underset{\lim }{\longrightarrow}\left\{Y_{\alpha}\right\}$ of Banach spaces, which is equipped with the uniform convergence over all ball $Y_{\alpha}$ topologies. It follows that $X^{\prime}$ is a complete polynormed space. Indeed, if $\left(f_{\lambda}\right)$ is a Cauchy net in $X^{\prime}$, then it converges to a linear functional $f: X \rightarrow \mathbb{C}$ with respect to the point-converges topology. Since $f_{\lambda} \mid$ ball $Y_{\alpha} \rightarrow f \mid$ ball $Y_{\alpha}$ uniformly, it follows that $f \mid Y_{\alpha} \in Y_{\alpha}^{*}$ for all $\alpha$. Hence $f \in X^{\prime}$. 
Theorem 2.1. Let $A$ be a multinormed $C^{*}$-algebra. Then $A$ is a multinormed $W^{*}$-algebra if and only if it is a bornological dual $X^{\prime}$ of a certain $\ell_{1}$-normed space $X$. In this case $X$ is unique up to an isometry.

Proof. By Proposition 2.1, $A$ is a multinormed $W^{*}$-algebra iff it is a central completion of a certain $W^{*}$-algebra $B$. Assume $A$ is a completion of $B$ with respect to the central topology determined by a family $\left(e_{\iota}\right)$ of its central projections such that $\vee_{\iota} e_{\iota}=1$. As above consider the complete bornological space $X=\bigcup_{\alpha} Y_{\alpha}$ in the predual $Y$ of $B$. By Lemma 2.4, $X$ is an $\ell_{1}$-normed space (see Definition 2.1). Furthermore, we have a well-defined linear mapping $\varphi: B \rightarrow X^{\prime}, \varphi(b)=b \mid X$. Since $\varphi(b)\left|Y_{\alpha}=b\right| Y_{\alpha} \in Y_{\alpha}^{*}$ for all $\alpha$, it follows that $\varphi(b) \in X^{\prime}$. By Lemma 2.4. $d_{\alpha}(\varphi(b))=\left\|\varphi(b)\left|Y_{\alpha}\|=\| b\right| Y_{\alpha}\right\|=\|b\|_{\alpha}$. Taking into account that $B$ is dense in $A$ and $X^{\prime}$ is complete, we derive that $\varphi$ is extended up to the embedding $\varphi: A \rightarrow X^{\prime}$, $d_{\alpha}(\varphi(a))=\|a\|_{\alpha}, \alpha \in \Lambda$. Actually, $\varphi(A)=X^{\prime}$. Indeed, take $f \in X^{\prime}$ and let $f_{\alpha}=f \mid Y_{\alpha}$ for each $\alpha$. Consider a bounded extension $b_{\alpha} \in Y^{*}$ of $f_{\alpha}$. Then $\left(b_{\alpha}\right)$ is a net in $B$. If $\iota \in \alpha \cap \beta$, then

$$
\left\|b_{\alpha}-b_{\beta}\right\|_{\iota}=d_{\iota}\left(\varphi\left(b_{\alpha}\right)-\varphi\left(b_{\beta}\right)\right)=\left\|b_{\alpha}\left|Y_{\iota}-b_{\beta}\right| Y_{\iota}\right\|=\left\|f\left|Y_{\iota}-f\right| Y_{\iota}\right\|=0 .
$$

Hence $\left(b_{\alpha}\right)$ is a Cauchy net in $A$. Put $a=\lim _{\alpha} b_{\alpha} \in A$. Then $\varphi(a)=\lim _{\alpha} \varphi\left(b_{\alpha}\right)$ in $X^{\prime}$. It follows that $\varphi(a) y_{\iota}=\lim _{\alpha, \iota \in \alpha} \varphi\left(b_{\alpha}\right) y_{\iota}=\lim _{\alpha, \iota \in \alpha} f_{\alpha}\left(y_{\iota}\right)=\lim _{\alpha, \iota \in \alpha} f\left(y_{\iota}\right)$ $=f\left(y_{\iota}\right)$ for all $y_{\iota} \in Y_{\iota}, \iota \in \Xi$. Hence $\varphi(a)=f$.

Now assume that $A=X^{\prime}$ for a certain $\ell_{1}$-normed space $X$. By Definition 2.1. there is a locally $\ell_{1}$-quotient mapping $\pi: \bigoplus_{\iota} E_{\iota} \rightarrow X$. Let $Y$ be the norm-completion of $X$ and let $Y_{\alpha}=\pi\left(\bigoplus_{\iota \in \alpha}^{1} E_{\iota}\right)$ be the closed subspaces in $Y$. Since $\pi: \bigoplus_{\iota \in \alpha}^{1} E_{\iota} \rightarrow Y_{\alpha}$ is the quotient mapping, it follows that $\pi_{\alpha}^{*}: Y_{\alpha}^{*} \rightarrow \bigoplus_{\iota \in \alpha}^{\infty} E_{\iota}^{*}$ is an isometry, where $\pi_{\alpha}=\pi \mid \bigoplus_{\iota \in \alpha}^{1} E_{\iota}$. By assumption $\left\{d_{\alpha}\right\}$ is a defining family of $C^{*}$-seminorms of $A$. Then for $a \in A$,

$$
d_{\alpha}(a)=\left\|a\left|Y_{\alpha}\|=\| \pi_{\alpha}^{*}\left(a \mid Y_{\alpha}\right)\left\|=\max _{\iota \in \alpha}\right\| \pi_{\iota}^{*}\left(a \mid Y_{\iota}\right)\left\|=\max _{\iota \in \alpha}\right\| a\right| Y_{\iota}\right\|=\max _{\iota \in \alpha} d_{\iota}(a),
$$

for all $\alpha$. Thus $\left\{d_{\iota}\right\}$ is a defining family of $C^{*}$-seminorms on $A$. Consider the linear mapping $\psi: Y^{*} \rightarrow B, \psi(f)=f \mid X$, where $B$ is the $C^{*}$-algebra of all bounded elements in $A$. If $f \in Y^{*}$, then $\sup _{\alpha} d_{\alpha}(\psi(f))=\sup _{\alpha}\left\|f \mid Y_{\alpha}\right\| \leq\|f\|=$ $\|f \mid X\| \leq \sup _{\alpha} d_{\alpha}(\psi(f))$, that is, $\psi(f) \in B$ and $\|\psi(f)\|=\|f\|$. If $b \in B$, then $b: X \rightarrow \mathbb{C}$ is norm bounded. Therefore it has a unique bounded extension $f \in Y^{*}$, that is, $\psi(f)=b$. Thus $\psi$ is an isometric isomorphism and $Y$ is the predual of the $C^{*}$-algebra $B$. In particular, $B$ is a $W^{*}$-algebra. Further, one can easily verify that $I_{\iota}=Y_{\iota}^{\perp}$. Then $I_{\iota}$ is a $\sigma\left(Y^{*}, Y\right)$-closed ideal in $B$. In particular, $I_{\iota}=B f_{\iota}$ for some central projection $f_{\iota}$. Using Lemma 2.1 we obtain that $d_{\iota}(b)=$ $d_{\iota}\left(\varphi_{\iota}(b)\right)=\left\|\varphi_{\iota}(b)\right\|$, where $\varphi_{\iota}: B \rightarrow B / I_{\iota}$ is the quotient $*$-homomorphism. Since $B=B e_{\iota} \oplus B f_{\iota}$, it follows that $\varphi_{\iota}: B e_{\iota} \rightarrow B / I_{\iota}$ is a $*$-isomorphism, where $e_{\iota}=1-f_{\iota}$. Therefore $\varphi_{\iota}: B e_{\iota} \rightarrow B / I_{\iota}$ is an isometry and $d_{\iota}(b)=\left\|\varphi_{\iota}(b)\right\|=\left\|b e_{\iota}\right\|=\|b\|_{\iota}$ for all $b \in B$. Hence $\left\{d_{\iota}\right\}$ is a family of $C^{*}$-seminorms associated with the central projections $\left(e_{\iota}\right)$ in $B$. Note that $\vee_{\iota} e_{\iota}=1$. Indeed, since $B \wedge_{\iota} f_{\iota}=\bigcap_{\iota} I_{\iota}=\bigcap_{\iota} Y_{\iota}^{\perp}=$ $\left(\sum_{\iota} Y_{\iota}\right)^{\perp}=\{0\}$, it follows that $\vee_{\iota} e_{\iota}=1-\wedge_{\iota} f_{\iota}=1$. Thus $A$ is a central completion of $B$. 
Finally, assume that $A=X^{\prime}=Z^{\prime}$ for some $\ell_{1}$-normed spaces $X$ and $Z$. Since their completions are the preduals of the $W^{*}$-algebra $B$, we conclude that $X$ and $Z$ are dense subspaces in the same Banach space $Y$ [25, 1.13.3], where $Y$ is the predual of $B$. Further, let $X=\bigcup_{\alpha} Y_{\alpha}$ and $Z=\bigcup_{v} Z_{v}$ be the relevant unions. By assumption, the families $\left\{d_{\alpha}\right\}$ and $\left\{d_{v}\right\}$ of $C^{*}$-seminorms on $A$ are equivalent. Then for each $\alpha$ there corresponds $v$ such that $d_{\alpha} \leq C_{\alpha v} d_{v}$ and vice versa. Thus $Z_{v}^{\perp}=I_{v} \subseteq I_{\alpha}=Y_{\alpha}^{\perp}$, which in turn implies that $Y_{\alpha} \subseteq Z_{v}$ (see Lemma 2.3). Hence $X=Z$.

\section{The DOMAIN OF A CENTRAL TOPOLOGY}

In this section we introduce local von Neumann algebras and prove a Sakai type theorem (see [25, 1.16.7]) for a multinormed $W^{*}$-algebra.

3.1. Noncommutative continuous functions. Let $H$ be a Hilbert space and let $\left(p_{\iota}\right)_{\iota \in \Xi}$ be a family of projections in $\mathcal{B}(H)$ such that $\vee_{\iota} p_{\iota}=1$. Thus $\mathcal{E}=\left\{p_{\alpha}\right.$ : $\alpha \in \Lambda\}$ is a quantum domain in $H$, where $p_{\alpha}=\vee_{\iota \in \alpha} p_{\iota}$ (see Section 11). Note that the commutant $\mathcal{E}^{\prime}$ in $\mathcal{B}(H)$ is a unital von Neumann algebra on $H$. The family $\|u\|_{\alpha}=\left\|u p_{\alpha}\right\|, u \in \mathcal{E}^{\prime}, \alpha \in \Lambda$ of $C^{*}$-seminorms defines a polynormed topology in $\mathcal{E}^{\prime}$. The completion of $\mathcal{E}^{\prime}$ with respect to this topology is denoted by $\mathcal{E}_{\mathcal{D}}^{\prime}$, where $\mathcal{D}$ indicates the union space $\bigcup_{\alpha} H_{\alpha}\left(H_{\alpha}=p_{\alpha}(H)\right)$ of the domain $\mathcal{E}$. Thus $\mathcal{E}_{\mathcal{D}}^{\prime}$ is a unital multinormed $C^{*}$-algebra. If $\mathcal{E}$ consists of mutually commuting projections, then we say that $\mathcal{E}$ is a domain in $H$.

Lemma 3.1. The algebra $\mathcal{E}_{\mathcal{D}}^{\prime}$ is a central completion of $\mathcal{E}^{\prime}$ associated with the uniquely defined central projections $e=\left(e_{\iota}\right), e_{\iota} \geq p_{\iota}, \iota \in \Xi$ in $\mathcal{E}^{\prime}$. In particular, $\mathcal{E}_{\mathcal{D}}^{\prime}$ is a multinormed $W^{*}$-algebra, which is identified with the algebra $C_{\mathcal{E}}^{*}(\mathcal{D})$ of all noncommutative continuous functions on $\mathcal{E}$.

Proof. First note that $\mathcal{E}_{\mathcal{D}}^{\prime}$ is identified with a unital subalgebra in $L(\mathcal{D})$. By its very definition, $\mathcal{E}_{\mathcal{D}}^{\prime}=\lim _{\longleftarrow}\left\{\mathcal{E}^{\prime} / I_{\alpha}\right\}$, where $I_{\alpha}=$ ker $\|\cdot\|_{\alpha} \subseteq \mathcal{E}^{\prime}$. In particular, each $u \in \mathcal{E}_{\mathcal{D}}^{\prime}$ is an equivalence class of a compatible family $\left(u_{\alpha}\right)$ from $\mathcal{E}^{\prime}$. We identify $u$ with the linear mapping $u \in L(\mathcal{D}), u(x)=u_{\alpha}(x)$ if $x \in H_{\alpha}$. If $x \in H_{\alpha} \cap H_{\beta}$, then $u_{\alpha}(x)=u_{\gamma} p_{\alpha}(x)=u_{\gamma} p_{\beta}(x)=u_{\beta}(x)$, where $\gamma=\alpha \cup \beta$. Moreover, if $\left(v_{\alpha}\right)$ is another compatible family representing $u$, then $u_{\alpha}=v_{\alpha}\left(\bmod I_{\alpha}\right)$ for all $\alpha$. Therefore $u_{\alpha}(x)=v_{\alpha}(x), x \in H_{\alpha}$. Thus $u$ is a well-defined linear transformation on $\mathcal{D}$. If $u(x)=0$ for all $x \in \mathcal{D}$, then $u_{\alpha} p_{\alpha}=0$, that is, $\|u\|_{\alpha}=0$ for all $\alpha$. Hence $u=0$. Thus $\mathcal{E}_{\mathcal{D}}^{\prime} \subseteq L(\mathcal{D})$.

Let us prove that $\mathcal{E}^{\prime}$ is the $C^{*}$-algebra of all bounded elements of $\mathcal{E}_{\mathcal{D}}^{\prime}$. Since $\sup _{\alpha}\|u\|_{\alpha} \leq\|u\|$, it follows that each $u \in \mathcal{E}^{\prime}$ is a bounded element of $\mathcal{E}_{\mathcal{D}}^{\prime}$. Conversely, if $b \in \mathcal{E}_{\mathcal{D}}^{\prime}$ is a bounded element, then $\|b(x)\|=\left\|b_{\alpha}(x)\right\| \leq\|b\|_{\alpha}\|x\| \leq$ $\sup _{\beta}\|b\|_{\beta}\|x\|$ for each $x \in H_{\alpha}$. Thus $b$ is bounded on $\mathcal{D}$, and it has a unique extension $b \in \mathcal{B}(H)$. If $x \in H_{\beta}$, then we have $p_{\alpha} b(x)=p_{\alpha} b_{\beta}(x)=b_{\beta} p_{\alpha}(x)=b p_{\alpha}(x)$ for all $\alpha$. Since $\mathcal{D}$ is dense in $H$ and $b$ is bounded, it follows that $p_{\alpha} b=b p_{\alpha}$ for all $\alpha$, that is, $b \in \mathcal{E}^{\prime}$.

Now consider the ideal $I_{\iota} \subseteq \mathcal{E}^{\prime}$. If $b=\lim _{\lambda} b_{\lambda}$ (SOT) of a certain net $\left(b_{\lambda}\right)$ in $I_{\iota}$, then $b p_{\iota}(x)=\lim _{\lambda} b_{\lambda} p_{\iota}(x)=0, x \in H$, that is, $b \in I_{\iota}$. Thus $I_{\iota}$ is a strongly closed ideal of the von Neumann algebra $\mathcal{E}^{\prime}$. Therefore $I_{\iota}=\mathcal{E}^{\prime} f_{\iota}$ for the uniquely determined projection $f_{\iota} \in \mathcal{E}^{\prime} \cap \mathcal{E}^{\prime \prime}$. Put $e_{\iota}=1-f_{\iota} \in \mathcal{E}^{\prime} \cap \mathcal{E}^{\prime \prime}$. The quotient homomorphism $\pi: \mathcal{E}^{\prime} e_{\iota} \rightarrow \mathcal{E}^{\prime} / I_{\iota}$ turns out to be a $*$-isomorphism. In particular, 
it is an isometry. Take $b \in \mathcal{E}^{\prime}$. Since $b=b e_{\iota}+b f_{\iota}$, we have $b p_{\iota}=b e_{\iota} p_{\iota}+b f_{\iota} p_{\iota}$, $b f_{\iota} \in I_{\iota}$. Then $0=\left\|b f_{\iota}\right\|_{\iota}=\left\|b f_{\iota} p_{\iota}\right\|$, that is, $b p_{\iota}=b e_{\iota} p_{\iota}$. Using Lemma 2.1, we derive that

$$
\|b\|_{\iota}=\left\|b p_{\iota}\right\|=\left\|b e_{\iota} p_{\iota}\right\|=\left\|b e_{\iota}\right\|_{\iota}=\left\|\pi\left(b e_{\iota}\right)\right\|_{\iota}=\left\|\pi\left(b e_{\iota}\right)\right\|=\left\|b e_{\iota}\right\| .
$$

In particular, $\|b\|_{\alpha}=\left\|b e_{\alpha}\right\|$ thanks to Lemma 2.3. Note that $\left\|p_{\alpha}-e_{\alpha} p_{\alpha}\right\|=$ $\left\|1-e_{\alpha}\right\|_{\alpha}=\left\|\left(1-e_{\alpha}\right) e_{\alpha}\right\|=0$, that is, $p_{\alpha} \leq e_{\alpha}$. In particular, $1=\vee_{\iota} e_{\iota}$. Thus the multinormed topology in $\mathcal{E}^{\prime}$ is just the central topology associated with the family of its central projections $\left(e_{\iota}\right)$. Using Proposition 2.1. we conclude that $\mathcal{E}_{\mathcal{D}}^{\prime}$ is a multinormed $W^{*}$-algebra.

Finally, by its very definition $C_{\mathcal{E}}^{*}(\mathcal{D})=\left\{T \in L(\mathcal{D}): p_{\alpha} T \subseteq T p_{\alpha}, T p_{\alpha} \in \mathcal{B}(H)\right.$, $\alpha \in \Lambda\}$ (see Section 1), which is a multinormed $C^{*}$-algebra equipped with the family of $C^{*}$-seminorms $\|T\|_{\alpha}^{\prime}=\left\|T p_{\alpha}\right\|, T \in C, \alpha \in \Lambda$ [5]. If $\alpha \subseteq \beta$, then the canonical mapping $\mathcal{E}^{\prime} / I_{\beta} \rightarrow \mathcal{E}^{\prime} / I_{\alpha}$ is reduced to the mapping $\mathcal{E}^{\prime} e_{\beta} \rightarrow \mathcal{E}^{\prime} e_{\alpha}, b \mapsto b e_{\alpha}$. Thus $\mathcal{E}_{\mathcal{D}}^{\prime}=\lim _{\longleftarrow}\left\{\mathcal{E}^{\prime} e_{\alpha}\right\}$. As we have noted above each $a=\left(a_{\alpha}\right)_{\alpha} \in \lim _{\longleftarrow}\left\{\mathcal{E}^{\prime} e_{\alpha}\right\}$ is identified with the linear mapping $a \in L(\mathcal{D}), a(x)=a_{\alpha}(x)$ if $x \in \overleftarrow{H_{\alpha}}$. If $x \in H_{\beta}$, then $p_{\alpha} a(x)=p_{\alpha} a_{\beta}(x)=a_{\beta} p_{\alpha}(x)=a p_{\alpha}(x)$, that is, $p_{\alpha} a \subseteq a p_{\alpha}$ for all $\alpha$. Hence $\mathcal{E}_{\mathcal{D}}^{\prime} \subseteq C_{\mathcal{E}}^{*}(\mathcal{D})$ and $\|a\|_{\alpha}=\left\|a_{\alpha}\right\|=\left\|a p_{\alpha}\right\|=\|a\|_{\alpha}^{\prime}, a \in \mathcal{E}_{\mathcal{D}}^{\prime}$ for all $\alpha$. The latter means that $\mathcal{E}_{\mathcal{D}}^{\prime}$ is a closed $*$-subalgebra in $C_{\mathcal{E}}^{*}(\mathcal{D})$.

Conversely, take a bounded element $T$ from $C_{\mathcal{E}}^{*}(\mathcal{D})$. Then $\sup _{\alpha}\left\|T p_{\alpha}\right\|<\infty$ and $T$ has the unique extension up to $T \in \mathcal{B}(H)$. But $p_{\alpha} T(x)=T p_{\alpha}(x)$ for all $x \in \mathcal{D}$. Hence $p_{\alpha} T=T p_{\alpha}$ in $\mathcal{B}(H)$, that is, $T \in \mathcal{E}^{\prime}$. Thus $\mathcal{E}_{\mathcal{D}}^{\prime}$ contains all bounded elements of $C_{\mathcal{E}}^{*}(\mathcal{D})$, which is dense in $C_{\mathcal{E}}^{*}(\mathcal{D})$ [24]. Hence $\mathcal{E}_{\mathcal{D}}^{\prime}=C_{\mathcal{E}}^{*}(\mathcal{D})$.

The following assertion generalizes the representation theorem for a multinormed $C^{*}$-algebra (see [18, [5]) on a quantum domain.

Proposition 3.1. Let $A$ be a multinormed $C^{*}$-algebra with its defining family of $C^{*}$-seminorms $\left\{\|\cdot\|_{\iota}: \iota \in \Xi\right\}$. There is a (commutative) domain $\mathcal{E}=\left\{e_{\alpha}: \alpha \in \Lambda\right\}$ in a Hilbert space $H$ and $*$-embedding $\varphi: A \hookrightarrow C_{\mathcal{E}}^{*}(\mathcal{D})$ such that $\|\varphi(a)\|_{\alpha}=\|a\|_{\alpha}$, $a \in A, \alpha \in \Lambda$, where $\mathcal{D}$ is the union space of $\mathcal{E}$.

Proof. Using [18] and [5], we conclude that $A$ is a closed $*$-subalgebra of $C_{\mathcal{E}}^{*}(\mathcal{D})$ for a certain quantum domain $\mathcal{E}=\left\{p_{\alpha}: \alpha \in \Lambda\right\}$ in a Hilbert space $H$, where $p_{\alpha}=\vee_{\iota \in \alpha} p_{\iota}$. Based on Lemma 3.1, we have another commutative domain $\mathcal{E}_{e}=\left\{e_{\alpha}: \alpha \in \Lambda\right\}$ in $H$ associated with the uniquely defined family of central projections $e=\left(e_{\iota}\right)$, $e_{\iota} \geq p_{\iota}, \iota \in \Xi$ in $\mathcal{E}^{\prime}$. Let $\mathcal{D}_{e}=\bigcup_{\alpha} e_{\alpha}(H)$ be its union space. Note that $\mathcal{D} \subseteq \mathcal{D}_{e}$. Since $\mathcal{E}_{e} \subseteq \mathcal{E}^{\prime \prime}$, it follows that $\mathcal{E}^{\prime}=\mathcal{E}^{\prime \prime \prime} \subseteq \mathcal{E}_{e}^{\prime}$ in $\mathcal{B}(H)$. Moreover, if $b \in \mathcal{E}^{\prime}$, then $\|b\|_{\alpha}=\left\|b p_{\alpha}\right\|=\left\|b e_{\alpha}\right\|$ thanks to Lemma 3.1. This means that the inclusion $\mathcal{E}^{\prime} \subseteq \mathcal{E}_{e}^{\prime}$ is compatible with the relevant central topologies. Using again Lemma 3.1 we conclude that $C_{\mathcal{E}}^{*}(\mathcal{D})=\mathcal{E}_{\mathcal{D}}^{\prime} \subseteq C_{\mathcal{E}_{e}}^{*}\left(\mathcal{D}_{e}\right)$. In particular, $A \subseteq C_{\mathcal{E}_{e}}^{*}\left(\mathcal{D}_{e}\right)$ is a closed *-subalgebra.

Using Lemma 3.1, we conclude that the strong operator topology (briefly, SOT) is well defined on $\mathcal{E}_{\mathcal{D}}^{\prime}$ or $C_{\mathcal{E}}^{*}(\mathcal{D})$ by means of the family $\left\{s_{x}: x \in \mathcal{D}\right\}$ of seminorms $s_{x}(T)=\|T x\|, T \in C_{\mathcal{E}}^{*}(\mathcal{D}), x \in \mathcal{D}$. If $x \in H_{\alpha}$, then $s_{x}(T)=\left\|T p_{\alpha} x\right\| \leq\|T\|_{\alpha}\|x\|$ for all $T \in C_{\mathcal{E}}^{*}(\mathcal{D})$; that is, SOT is weaker than the original multinormed topology. If $A$ is a subalgebra in $C_{\mathcal{E}}^{*}(\mathcal{D})$, then $A^{\prime}$ denotes the commutant of $A$ in $C_{\mathcal{E}}^{*}(\mathcal{D})$ as usual. Note that $A^{\prime}$ is strongly closed. Indeed, if $u=\lim _{t} u_{t}(\mathrm{SOT}),\left(u_{t}\right) \subseteq A^{\prime}$ and $x \in H_{\alpha}$, then $a u(x)=a_{\alpha} u(x)=\lim _{t} a_{\alpha} u_{t}(x)=\lim _{t} u_{t} a_{\alpha}(x)=u a(x)$ for all $a \in A$. Note that $\operatorname{span}\left\{p_{\alpha}: \alpha \in \Lambda\right\}^{-}(\mathrm{SOT}) \subseteq C_{\mathcal{E}}^{*}(\mathcal{D})^{\prime}$. 
3.2. Local von Neumann algebras. Now let $\mathcal{E}=\left\{p_{\alpha}: \alpha \in \Lambda\right\}$ be a (commutative) domain in a Hilbert space $H$ with its union space $\mathcal{D}$. By Lemma $3.1 C_{\mathcal{E}}^{*}(\mathcal{D})$ is a central completion of the commutant $\mathcal{E}^{\prime}$ in $\mathcal{B}(H)$. Moreover, $\|T\|_{\alpha}=\left\|T p_{\alpha}\right\|$, $T \in C_{\mathcal{E}}^{*}(\mathcal{D}), \alpha \in \Lambda$ is a defining family of $C^{*}$-seminorms on $C_{\mathcal{E}}^{*}(\mathcal{D})$. If $T \in C_{\mathcal{E}}^{*}(\mathcal{D})$, then we also write $T=\left(T_{\alpha}\right)$, where $T_{\alpha}=T \mid H_{\alpha}, \alpha \in \Lambda$. If $A$ is a $*$-subalgebra in $C_{\mathcal{E}}^{*}(\mathcal{D})$, then we put $A_{\alpha}=\left\{T_{\alpha}: T \in A\right\} \subseteq \mathcal{B}\left(H_{\alpha}\right)$.

Lemma 3.2. Let $A$ be a closed *-subalgebra in $C_{\mathcal{E}}^{*}(\mathcal{D})$. The *-subalgebra $A p_{\alpha}$ equipped with the $C^{*}$-seminorm $\|\cdot\|_{\alpha}$ turns out to be a $C^{*}$-algebra, which is *isomorphic to $A_{\alpha}$. Moreover, if $A p_{\alpha} \subseteq A$ and $A$ is strongly closed, then so is $A p_{\alpha}$.

Proof. As we know (see [27, [24, Corollary 1.12]), $A /$ ker $\|\cdot\|_{\alpha}$ is a $C^{*}$-algebra with respect to the induced $C^{*}$-norm. But the mapping $A / \operatorname{ker}\|\cdot\|_{\alpha} \rightarrow A_{\alpha}, T^{\sim} \mapsto T_{\alpha}$ is a $*$-isomorphism. Hence $A_{\alpha}$ is a $C^{*}$-subalgebra in $\mathcal{B}\left(H_{\alpha}\right)$. Since $A p_{\alpha} \rightarrow A_{\alpha}$, $T p_{\alpha} \mapsto T_{\alpha}$, is a $*$-isomorphism, it follows that $A p_{\alpha}$ is a $C^{*}$-algebra.

Finally, assume that $A$ is strongly closed and $A p_{\alpha} \subseteq A$. If $\lim _{\lambda} a_{\lambda} p_{\alpha}=b$ (SOT) for a certain net $\left(a_{\lambda}\right)$ in $A$, then $b \in A$ and $b p_{\alpha}(x)=\lim _{\lambda} a_{\lambda} p_{\alpha}\left(p_{\alpha}(x)\right)=b(x)$, $x \in \mathcal{D}$. Thus $b=b p_{\alpha} \in A p_{\alpha}$.

Let us introduce local von Neumann algebras on $\mathcal{D}$.

Definition 3.1. A $*$-subalgebra $A \subseteq C_{\mathcal{E}}^{*}(\mathcal{D})$ is said to be a local von Neumann algebra on $\mathcal{D}$ if it is strongly closed and $A p_{\alpha} \subseteq A$ for all $\alpha$.

If $A$ is a $*$-subalgebra in $C_{\mathcal{E}}^{*}(\mathcal{D})$, then its commutant $A^{\prime}$ in $C_{\mathcal{E}}^{*}(\mathcal{D})$ is a local von Neumann algebra on $\mathcal{D}$. Indeed, since $\mathcal{E} \subseteq A^{\prime}$, we conclude that $A^{\prime} p_{\alpha} \subseteq A^{\prime}$ for all $\alpha$. Moreover, $A^{\prime}$ is strongly closed.

Lemma 3.3. If $A$ is a local von Neumann algebra on $\mathcal{D}$, then it has a unit and the algebra $A$ is identified with a unital local von Neumann subalgebra in $C_{\mathcal{S}}^{*}(\mathcal{O})$ for another domain $\mathcal{S}$ with its union space $\mathcal{O}, \mathcal{O} \subseteq \mathcal{D}$.

Proof. Since $A$ is strongly closed, it follows that $A$ is a closed $*$-subalgebra in $C_{\mathcal{E}}^{*}(\mathcal{D})$. Using [18, Theorem 2.6], we conclude that there is an approximate unit $\left(u_{t}\right)$ in $A$. Actually, it can be assumed that $\left(u_{t}\right)$ is an approximate unit of the $C^{*}$-algebra $\mathfrak{b}(A)$ (see [24, Corollary 3.12]). Note that $\mathfrak{b}(A)=\left\{T \in A:\|T\|=\sup _{\alpha}\|T\|_{\alpha}<\infty\right\}$ and it is canonically identified with the $C^{*}$-subalgebra of $\mathcal{B}(H)$. Then $\lim _{t} u_{t}=q$ (SOT) in $\mathcal{B}(H)$, where $q$ is selfadjoint. But $u_{t} p_{\alpha}=p_{\alpha} u_{t}$ for all $\alpha \in \Lambda$. Then

$$
p_{\alpha} q(x)=\lim _{t} p_{\alpha} u_{t}(x)=\lim _{t} u_{t} p_{\alpha}(x)=q p_{\alpha}(x)
$$

for all $x \in \mathcal{D}$, that is, $q(\mathcal{D}) \subseteq \mathcal{D}$ and $p_{\alpha} q \mid \mathcal{D} \subseteq q p_{\alpha}$. Hence $q \mid \mathcal{D} \in C_{\mathcal{E}}^{*}(\mathcal{D})$. Put $q=q \mid \mathcal{D}$. Then $q \in A$, for $A$ is strongly closed. If $a \in A$ and $x \in \mathcal{D}$, then $q a(x)=\lim _{t} u_{t} a(x)$. But $\lim _{t} u_{t} a=a$ in $C_{\mathcal{E}}^{*}(\mathcal{D})$. In particular, $\lim _{t} u_{t} a(x)=$ $a(x), x \in \mathcal{D}$. Thus $q a=a$ for all $a \in A$, that is, $q$ is a unit of $A$. Moreover, $q_{\alpha}=q p_{\alpha} \in A p_{\alpha} \subseteq A, \alpha \in \Lambda$ (see Definition 3.1), and $q_{\alpha}$ is a projection such that $q_{\alpha} \leq p_{\alpha}$. Consider the quantum domain $\mathcal{S}=\left\{q_{\alpha}: \alpha \in \Lambda\right\}$ in $q(H)$ with its union space $\mathcal{O}$. Actually, it is a domain, for $\mathcal{S}$ is commutative. Moreover, $\mathcal{O} \subseteq \mathcal{D}$ and we have a well-defined topological $*$-isomorphism $q C_{\mathcal{E}}^{*}(\mathcal{D}) q \rightarrow C_{\mathcal{S}}^{*}(\mathcal{O}), u \mapsto u \mid \mathcal{O}$. If $u=q T q$, then $\|u \mid \mathcal{O}\|_{\alpha}=\left\|u q_{\alpha}\right\|=\left\|u p_{\alpha}\right\|=\|u\|_{\alpha}$ for all $\alpha \in \Lambda$. The range of $A$ by means of this mapping is denoted by $A_{q}$. If $x \in \mathcal{D}$, then $s_{x}(T)=\|T x\|=$ $\|T q(x)\|=s_{q(x)}(T \mid \mathcal{O})$ for all $T \in q C_{\mathcal{E}}^{*}(\mathcal{D}) q$. It follows that $A_{q}$ is strongly closed in $C_{\mathcal{S}}^{*}(\mathcal{O})$ and $q \mid \mathcal{O}=1_{\mathcal{O}}$. 
The following assertion shows that a local von Neumann algebra is a direct limit of its von Neumann subalgebras.

Proposition 3.2. Let $A$ be a local von Neumann algebra on $\mathcal{D}$, and let $S=$ $\bigcup_{\alpha} A p_{\alpha}$. Then $A$ is a multinormed $W^{*}$-algebra and $A=S^{-}(S O T)$ in $C_{\mathcal{E}}^{*}(\mathcal{D})$. Moreover, $\mathfrak{b}(A)$ is a von Neumann algebra on $H$ and $\mathfrak{b}(A)=S^{-}(S O T)$ in $\mathcal{B}(H)$. Thus

$$
A=\lim _{\longrightarrow}\left\{A p_{\alpha}\right\} \quad \text { on } \mathcal{D} .
$$

Proof. Put $B=\mathfrak{b}(A)$. Using Lemma 3.3, we can assume that $1_{\mathcal{D}} \in A$. Then $p_{\alpha}=1_{\mathcal{D}} p_{\alpha} \in A p_{\alpha} \subseteq A$ for all $\alpha \in \Lambda$. By Lemma 3.2, each $A p_{\alpha}$ is strongly closed and $S$ being the union $\bigcup_{\alpha} A p_{\alpha}$ of its upward filtered family of $*$-subalgebras is a *-subalgebra in $B$. In particular, each $A_{\alpha}$ is a unital von Neumann algebra on $H_{\alpha}$. We know that $A=\lim \left\{A p_{\alpha}\right\}$ (see Lemma 3.2). If $\alpha \subseteq \beta$ we have a well-defined connecting $*$-homomorphism $\varphi_{\alpha \beta}: A p_{\beta} \rightarrow A p_{\alpha}, \varphi_{\alpha \beta}(a)=a p_{\alpha}$ of von Neumann algebras, which is weak* continuous (see [25, 1.7.7]). Hence $A$ is a multinormed $W^{*}$-algebra. Using Lemma 2.2 , we conclude that $B$ is strongly closed in $\mathcal{B}(H)$.

If $b \in B$, then $b=\lim _{\alpha} b p_{\alpha}(\mathrm{SOT})$ in $\mathcal{B}(H)$. Hence $B=S^{-}(\mathrm{SOT})$ in $\mathcal{B}(H)$. Further, take $a \in A$ and $x \in H_{\beta}$. Then $\lim _{\alpha} s_{x}\left(a-a p_{\alpha}\right)=\lim _{\alpha, \alpha \supseteq \beta} \| a p_{\beta}(x)-$ $a p_{\alpha} p_{\beta}(x) \|=0$. Hence $A=S^{-}(\mathrm{SOT})$ in $C_{\mathcal{E}}^{*}(\mathcal{D})$.

Corollary 3.1. Let $A \subseteq C_{\mathcal{E}}^{*}(\mathcal{D})$ be a $*$-subalgebra such that $1_{\mathcal{D}} \in A$. Then $A$ is a local von Neumann algebra on $\mathcal{D}$ if and only if $A=A^{\prime \prime}$.

Proof. If $A=A^{\prime \prime}$, then $A$ being the commutant of a $*$-subalgebra turns out to be a local von Neumann algebra. Conversely, assume that $A$ is a local von Neumann algebra on $\mathcal{D}$. Using Proposition 3.2 , we conclude that $B$ is a unital von Neumann algebra on $H$, where $B=\mathfrak{b}(A)$. Note that $A^{\prime}=B^{\prime}$ in $C_{\mathcal{E}}^{*}(\mathcal{D})$. Indeed, if $u \in B^{\prime}$ and $a \in A$, then $a=\lim _{\lambda} b_{\lambda}$ in $C_{\mathcal{E}}^{*}(\mathcal{D})$ for a certain net $\left(b_{\lambda}\right)$ in $B$, and $u a=\lim _{\lambda} u b_{\lambda}=$ $\lim _{\lambda} b_{\lambda} u=a u$. Hence $A^{\prime}=B^{\prime}$. For a while let us use the notation $C^{\sim}$ for the commutant in $\mathcal{B}(H)$ of a subset $C \subseteq \mathcal{B}(H)$. Note that $\mathfrak{b}\left(B^{\prime}\right)=B^{\sim}$. Indeed, if $T \in B^{\sim}$, then $T \in \mathcal{E}^{\sim} \subseteq C_{\mathcal{E}}^{*}(\mathcal{D})$, for $\mathcal{E} \subseteq B$ (see Definition 3.1). Hence $T \in B^{\prime}$.

Take $u \in \mathfrak{b}\left(A^{\prime \prime}\right)$. Then $u$ commutes with all elements from $B^{\prime}$, in particular from $\mathfrak{b}\left(B^{\prime}\right)$, that is, $u \in B^{\sim \sim}$. But $B^{\sim \sim}=B$ thanks to the bicommutant theorem [23, Theorem 4.1.5]. Therefore $u \in B$. Hence $\mathfrak{b}\left(A^{\prime \prime}\right) \subseteq B \subseteq A$. But $\mathfrak{b}\left(A^{\prime \prime}\right)$ is dense in $A^{\prime \prime}$. So, $A^{\prime \prime}=A$.

The bicommutant theorem is not true in the general case of a strongly closed *-subalgebra.

Example. Let $\mathcal{E}=\left\{p_{n}: n \in \mathbb{N}\right\}$ be a countable domain and let $A=\left\{\lambda 1_{\mathcal{D}}+\mu p_{1}\right.$ : $\lambda, \mu \in \mathbb{C}\}$ be the unital 2-dimensional $*$-subalgebra in $C_{\mathcal{E}}^{*}(\mathcal{D})$. If $T=\lim _{t}\left(\lambda_{t} 1_{\mathcal{D}}+\right.$ $\left.\mu_{t} p_{1}\right)(\mathrm{SOT})$ for a certain net $\left(\lambda_{t} 1_{\mathcal{D}}+\mu_{t} p_{1}\right)$ in $A$, then $T x=\lim _{t} \lambda_{t} x$ for each unit vector $x \in \mathcal{D} \cap H_{1}^{\perp}$. Hence there is $\lambda=\lim _{t} \lambda_{t}$ and $T x=\lambda x$. If $y$ is a unit vector in $H_{1}$, then $\lim _{t} \mu_{t} y=\lim _{t} \mu_{t} p_{1}(y)=T y-\lambda y$; that is, there is $\mu=\lim _{t} \mu_{t}$. Thus $T=\lambda 1_{\mathcal{D}}+\mu p_{1} \in A$. So, $A$ is a strongly closed $*$-subalgebra in $C_{\mathcal{E}}^{*}(\mathcal{D})$. But $A \neq A^{\prime \prime}$, for $p_{2} \in A^{\prime \prime} \backslash A$.

That was a serious gap of the papers [20] and [21].

Corollary 3.2. If $Z\left(C_{\mathcal{E}}^{*}(\mathcal{D})\right)$ is the center of the algebra $C_{\mathcal{E}}^{*}(\mathcal{D})$, then $Z\left(C_{\mathcal{E}}^{*}(\mathcal{D})\right)=$ $\operatorname{alg}(\mathcal{E})^{-}(S O T)$, where $\operatorname{alg}(\mathcal{E})$ is the unital algebra generated by $\mathcal{E}$. 
Proof. Assume $A=\operatorname{alg}(\mathcal{E})$. Note that $A$ consists of all polynomials $\sum_{\theta} \lambda_{\theta} e_{\theta}$, where $e_{\theta}=e_{\alpha_{1}} \cdots e_{\alpha_{n}}, \lambda_{\theta} \in \mathbb{C}$. In particular, $A$ is a unital $*$-subalgebra in $C_{\mathcal{E}}^{*}(\mathcal{D})$ and its SOT closure $A^{-}$is a local von Neumann algebra on $\mathcal{D}$. Moreover, $A^{\prime}=\mathcal{E}^{\prime}$ and $A \subseteq Z\left(C_{\mathcal{E}}^{*}(\mathcal{D})\right)$. Since $Z\left(C_{\mathcal{E}}^{*}(\mathcal{D})\right)$ is strongly closed, we conclude that $A^{-} \subseteq$ $Z\left(C_{\mathcal{E}}^{*}(\mathcal{D})\right)$. If $a \in Z\left(C_{\mathcal{E}}^{*}(\mathcal{D})\right)$ and $b \in \mathcal{E}^{\prime}$, then $a b=b a$, that is, $a \in \mathcal{E}^{\prime \prime}=A^{\prime \prime}$. Using Corollary 3.1, we conclude that $a \in A^{-}$. Hence $Z\left(C_{\mathcal{E}}^{*}(\mathcal{D})\right)=A^{-}$.

Corollary 3.3. If $A$ is a local von Neumann algebra, then it is a closed linear span of its projections. Moreover, if $a \in A$ with its polar decomposition $a=u|a|$, then $u \in A$.

Proof. A similar result for von Neumann algebras is well known [23, Ch. 4]. In particular, $\mathfrak{b}(A)$ is a closed linear span of its projections (see Proposition 3.2). But the norm topology in $\mathfrak{b}(A)$ is stronger than the original polynormed topology from $A$. Hence the linear span of projections is dense in $\mathfrak{b}(A)$. But $\mathfrak{b}(A)$ itself is dense in $A$ (see also [24, Proposition $1.11(4)]$ ). Therefore the linear span of projections is dense in $A$ too.

Finally, if $a=u|a|$, then $a p_{\alpha}=u p_{\alpha}\left|a p_{\alpha}\right|$ is the polar decomposition of $a p_{\alpha}$ in the von Neumann algebra $A p_{\alpha}$. Then $u p_{\alpha} \in A p_{\alpha}$ for all $\alpha$. But $u=\lim _{\alpha} u p_{\alpha}$ (SOT); therefore $u \in A$.

3.3. Sakai type theorem for multinormed $W^{*}$-algebras. Now we prove a Sakai type theorem for multinormed $W^{*}$-algebras classifying them as local von Neumann algebras on domains.

Theorem 3.1. Let $A$ be a multinormed $W^{*}$-algebra with its upward filtered family $\left\{\|\cdot\|_{\alpha}: \alpha \in \Lambda\right\}$ of $C^{*}$-seminorms. There exists a domain $\mathcal{E}=\left\{p_{\alpha}: \alpha \in \Lambda\right\}$ in a Hilbert space $H$ and $*$-isomorphism $A \rightarrow C_{\mathcal{E}}^{*}(\mathcal{D})$ onto a local von Neumann subalgebra on $\mathcal{D}$ such that $\|a\|_{\alpha}=\left\|a p_{\alpha}\right\|, a \in A$ for all $\alpha$.

Proof. By Proposition 2.1. we can assume that $A$ is a central completion of a $W^{*}$-algebra $B$ associated with a family $\left\{e_{\iota}: \iota \in \Xi\right\}$ of its central projections such that $\vee_{\iota} e_{\iota}=1$. Using the Sakai theorem [25, 1.16.7] (see also [25, 1.15.1]), we can assume that $B$ is a unital von Neumann algebra on a Hilbert space $H$ up to a *-isomorphism. In particular, $\mathcal{E}=\left\{e_{\alpha}: \alpha \in \Lambda\right\}$ is a domain in $H$ and $B \subseteq \mathcal{E}^{\prime}$. Note that the central topology in $\mathcal{E}^{\prime}$ associated with $\left(e_{\iota}\right)$ is reduced to the original central topology in $B$. The central completion $\mathcal{E}_{\mathcal{D}}^{\prime}$ of $\mathcal{E}^{\prime}$ is the $*$-algebra $C_{\mathcal{E}}^{*}(\mathcal{D})$ thanks to Lemma 3.1 where $\mathcal{D}$ is the union space of the domain $\mathcal{E}$. Since $C_{\mathcal{E}}^{*}(\mathcal{D})$ is complete, it follows that $A$ is just the closure of $B$ in $C_{\mathcal{E}}^{*}(\mathcal{D})$ (see Proposition 3.1). It remains to prove that $A$ is a local von Neumann subalgebra in $C_{\mathcal{E}}^{*}(\mathcal{D})$. Take $a \in A$. Then $a=\lim _{\lambda} a_{\lambda}$ (in $A$ ) for a certain net $\left(a_{\lambda}\right)$ in $B$. Thus $\lim _{\lambda}\left\|a-a_{\lambda}\right\|_{\alpha}=0$ for each $\alpha \in \Lambda$. In particular, $\lim _{\lambda}\left\|a e_{\alpha}-a_{\lambda} e_{\alpha}\right\|=\lim _{\lambda}\left\|a-a_{\lambda}\right\|_{\alpha}=0$ in $\mathcal{B}(H)$. But $a_{\lambda} e_{\alpha} \in B$, for $a_{\lambda}, e_{\alpha} \in B$. Since $B$ is closed in $\mathcal{B}(H)$, it follows that $a e_{\alpha} \in B$. Hence $A e_{\alpha} \subseteq B \subseteq A$ for all $\alpha \in \Lambda$.

Now assume that $a=\lim _{\lambda} a_{\lambda}(\mathrm{SOT})$ in $C_{\mathcal{E}}^{*}(\mathcal{D})$ for a certain net $\left(a_{\lambda}\right)$ in $A$. Fix $\alpha \in \Lambda$. Then $\lim _{\lambda} s_{x}\left(a-a_{\lambda}\right)=0$ for all $x \in H_{\alpha}$. Hence $a e_{\alpha}=\lim _{\lambda} a_{\lambda} e_{\alpha}$ (SOT) in $\mathcal{B}(H)$. But all $a_{\lambda} e_{\alpha} \in B$ and $B$ is strongly closed. Then $a e_{\alpha} \in B \subseteq A$. Further, let us prove that $a=\lim _{\alpha} a e_{\alpha}$ in $C_{\mathcal{E}}^{*}(\mathcal{D})$. For each $\beta \in \Lambda$, we have $\lim _{\alpha}\left\|a-a e_{\alpha}\right\|_{\beta}=\lim _{\alpha, \alpha \supseteq \beta}\left\|a-a e_{\alpha}\right\|_{\beta}=\lim _{\alpha, \alpha \supseteq \beta}\left\|a e_{\beta}-a e_{\alpha} e_{\beta}\right\|=0$. But $A$ is closed in $C_{\mathcal{E}}^{*}(\mathcal{D})$. Hence $a \in A$; that is, $A$ is strongly closed. Thus $A$ is a local von Neumann algebra on $\mathcal{D}$ (see Definition 3.1). 
Corollary 3.4. Let $A$ be a multinormed $C^{*}$-algebra. The following conditions are equivalent:

(i) $A$ is a multinormed $W^{*}$-algebra;

(ii) $A$ is a central completion of a $W^{*}$-algebra;

(iii) $A$ is the bornological dual $X^{\prime}$ of a uniquely (up to an isometry) defined $\ell_{1}$-normed space $X$;

(iv) $A$ is a local von Neumann algebra on the union space $\mathcal{D}$ of a (commutative) domain $\mathcal{E}$.

Proof. It suffices to use Proposition 2.1, Theorems 2.1 and 3.1.

Example. In the theory of quantum (or local operator) spaces [11, [12, [5] local von Neumann algebras appear in the following way (see [10, [8], [9]). Fix a set $J$ with its partition $J=\vee_{\kappa \in \Xi} J_{\kappa}$. We can identify $J$ with the family $\left\{J_{\kappa}\right\}$ of sets. Assume for each $w \in J$ there corresponds an atomic algebra $M_{n_{w}}$ of all scalar $n_{w}$-square matrices, where $n_{w}$ can be thought of as a value of a certain function $n: J \rightarrow \mathbb{N}$ at the point $w$. Then for each member $J_{\kappa}$ of the family $J$ is associated the von Neumann algebra $M_{J_{\kappa}}=\bigoplus_{w \in J_{\kappa}}^{\infty} M_{n_{w}}$. Consider the direct product $\mathfrak{D}_{J}=\prod_{\kappa \in \Xi} M_{J_{\kappa}}$, which is a multinormed $W^{*}$-algebra. By Theorem 3.1. $\mathfrak{D}_{J}$ is a local von Neumann algebra. Note that $\mathfrak{D}_{J} \subseteq C_{\mathcal{E}}^{*}(\mathcal{D})$, where $\mathcal{E}=\left\{H_{\alpha}: \alpha \in \Lambda\right\}$ is a domain in the Hilbert space $H=\bigoplus_{w \in J} \mathbb{C}^{n_{w}}$ and $H_{\alpha}=\bigoplus_{\kappa \in \alpha} N_{\kappa}, N_{\kappa}=\bigoplus_{w \in J_{\kappa}} \mathbb{C}^{n_{w}}$. So, we deal with the orthogonal family $\left(p_{\kappa}\right)$ of projections in $\mathcal{B}(H)$ such that $\sum_{\kappa} p_{\kappa}=1$. The predual of $\mathfrak{D}_{J}$ can easily be found. It is the direct sum $\mathcal{T}_{J}=\bigoplus_{\kappa \in \Xi} \mathcal{T}_{J_{\kappa}}$ in the space $\mathcal{T}(H)$ of all trace class operators on $H$, where each $\mathcal{T}_{J_{\kappa}}=\bigoplus_{w \in J_{\kappa}}^{1} \mathcal{T}_{n_{w}}$ is the operator space of all trace class matrices in $M_{J_{\kappa}}$ [10. Note that $\mathcal{T}_{J}$ is an $\ell_{1}$-normed space (see Definition 2.11). By Theorem 2.1, the space $\mathcal{T}_{J}$ is unique up to an isometry. Moreover, in this case, the bornological dual $\mathcal{T}_{J}^{\prime}$ is just the strong dual $\left(\mathcal{T}_{J}\right)_{\beta}^{\prime}$ of the polynormed direct sum $\mathcal{T}_{J}=\bigoplus_{\kappa \in \Xi} \mathcal{T}_{J_{\kappa}}$. Hence $\left(\mathcal{T}_{J}\right)_{\beta}^{\prime}=\mathfrak{D}_{J}$. The algebra $\mathfrak{D}_{J}$ plays the main role in the representation theorem and dual realization problem for quantum spaces [10].

\section{ACKNOWLEDGEMENTS}

The author thanks M. Fragoulopoulou for useful discussions and for sending the paper [13. The author also thanks M. Joiţa for sending the papers [20], [21], and the referee(s) for the proposed comments which improved the paper significantly.

\section{REFERENCES}

[1] Antoine J.-P., Inoue A., Trapani C., Partial *-algebras and their operator realizations, Math. Appl. 533, Kluwer Acad. Publ., Dordrecht (2002). MR.1947892 (2005d:47116)

[2] Bagarello F., Inoue A., Trapani C., Bicommutant of reduced unbounded operators, Proc. Amer. Math. Soc., 137 (2009) 3709-3716. MR2529878 (2010h:47148)

[3] Blecher D. P., The standard dual of an operator space, Pacific J. Math., 153 (1) (1992) 15-30. MR.1145913 (93d:47083)

[4] Conway J. B., A course in functional analysis, Grad. Texts in Math., Springer (1990). MR $1070713(91 \mathrm{e}: 46001)$ 
[5] Dosiev A. A., Local operator spaces, unbounded operators and multinormed $C^{*}$-algebras, J. Funct. Anal., 255 (2008) 1724-1760. MR2442081 (2010a:46133)

[6] Dosiev A. A., Quantized moment problem, Comptes Rendus Math., 344 (1) (2007) 627-630. MR2334073 (2008f:46086)

[7] Dosi A. A., Local operator algebras, fractional positivity and quantum moment problem, Trans. Amer. Math. Soc., 363 (2) (2011) 801-856. MR.2728586

[8] Dosi A. A., Noncommutative Mackey theorem, Inter. J. Math., 22 (4) (2011) 535-544. MR.2794460

[9] Dosi A. A., Quotients of quantum bornological spaces, Taiwan. J. Math., 15 (3) (2011), 12871303. MR2829912

[10] Dosi A. A., Quantum duality, unbounded operators and inductive limits, J. Mathematical Physics, 51 (6) (2010) 1-43. MR2676488

[11] Effros E. G., Webster C., Operator analogues of locally convex spaces, Operator Algebras and Applications (Samos 1996) (NATO Adv. Sci. Inst. Ser. C Math. Phys. Sci., 495), Kluwer (1997). MR 1462680(99b:46081)

[12] Effros E. G., Winkler S., Matrix convexity: operator analogues of the bipolar and HahnBanach theorems, J. Funct. Anal., 144 (1997) 117-152. MR1430718 (98e:46066)

[13] Fragoulopoulou M., On locally $W^{*}$-algebras, Yokohama Math. J., 34 (1-2) (1986) 35-51. MR886056 (88g:46084)

[14] Fragoulopoulou M., Topological algebras with involution, Math. Stud., 200 (2005). MR2172581 (2006m:46067)

[15] Harti R., Lukacs G., Bounded and unitary elements in pro-C ${ }^{*}$-algebras, Appl. Categor. Struct., 14 (2006) 151-164. MR2247450 (2007b:46134)

[16] Helemskii A. Ya., Banach and polynormed algebras: General theory, representations, homology, Nauka, Moscow (1989). MR1031991 (91h:46001)

[17] Hogbe-Nlend H., Bornologies and functional analysis: Introductory course on the theory of duality topology-bornology and its use in functional analysis, Elsevier (1977). MR0500064 (58:17774)

[18] Inoue A., Locally $C^{*}$-algebras, Memoirs Fac. Scien. Kyushu Univ. Ser. A, 25 (2) (1971) 197235. MR0305089 (46:4219)

[19] Inoue A., Tomita-Takesaki Theory in Algebras of Unbounded Operators, Lect. Notes Math. 1699, Springer (1998). MR1725388(2000j:47129)

[20] Joiţa M., Locally von Neumann algebras I, Bull. Math. Soc. Sci. Math. Roumanie, 42 (90) (1999) 51-64. MR1880655 (2002k:46157)

[21] Joiţa M., Locally von Neumann algebras II, Rendconti d. Cir. Matem. di Palermo, LI (II) (2002) 83-94. MR.1905709 (2003e:46107)

[22] Kutateladze S. S., Fundamentals of functional analysis, Kluwer Acad. Publ. 12 (1995). MR $1384068(97 \mathrm{a}: 46001)$

[23] Murphy G. J., $C^{*}$-algebras and operator theory, Acad. Press, Inc. (1990). MR.1074574 (91m:46084)

[24] Phillips N. C., Inverse limits of $C^{*}$-algebras, J. Operator Theory, 19 (1988) 159-195. MR.950831 (90c:46090)

[25] Sakai S., $C^{*}$-algebras and $W^{*}$-algebras, Springer-Verlag (1971). MR0442701 (56:1082)

[26] Schaefer H., Topological vector spaces, Springer-Verlag (1970). MR 0342978 (49:7722)

[27] Schmüdgen K., Über LMC ${ }^{*}$-Algebren, Math. Nachr., 68 (1975) 167-182. MR0394227 (52:15030)

[28] Schmüdgen K., Unbounded operator algebras and representation theory, Birkhäuser Verlag (1990). MR1056697 (91f:47062)

Middle East Technical University northern Cyprus Campus, Guzelyurt, KKTC, MERsin 10, TURKEY

E-mail address: dosiev@yahoo.com

E-mail address: dosiev@metu.edu.tr

$U R L:$ http://www.math.ncc.metu.edu.tr/content/members-dosiev.php 